\title{
Geostatistical Analysis of Mineral Deposit Associations using Soil, Stream Sediment, and Vein Geochemical Data from Kaiama, Northcentral Nigeria
}

\author{
V. C. Alepa ${ }^{1}$, S. A. Alimi ${ }^{1}$, A. P Babatunde ${ }^{1}$, T. W. Andongma ${ }^{2}$, D. S. Bonde ${ }^{3 *}$ \\ ${ }^{1}$ Department of Geology and Mineral Sciences, University of Ilorin, Ilorin, Nigeria \\ ${ }^{2}$ Department of Geology, Kano University of Science and Technology, Wundil, Kano State, Nigeria \\ ${ }^{3}$ Department of Physics, Kebbi State University of Science and Technology, Aliero, Kebbi State, Nigeria
}

\begin{tabular}{ll}
\hline DOI:10.36348/SJET.2019.v04i09.003 & | Received: 05.09.2019| Accepted: 13.09.2019| Published: 20.09 .2019 \\
*Corresponding author: Bonde Danladi Senchi &
\end{tabular}

\section{Abstract}

The application of geostatistics to data obtained from mineral prospecting can provide useful information for evaluating mineralization potential. The objectives of this study were to evaluate the spatial distribution of elements, establish relationships and understand chemical associations during mineral paragenesis in the studied area of Kaiama, Northcentral Nigeria. Major Oxide and Trace element analysis were carried out on 17 samples collected from three geologic media which are stream sediments, soils, and veins. The Geochemical results were subjected to multivariate statistical treatments in the form of correlation matrix (CM), Factor analysis (PCA), along with hierarchical cluster analysis (HCA) in order to classify the elements into groups that may signify common origin. All multivariate statistical tests were performed using SPSS (PASW Statistics 18) for Windows. The trace element geochemical composition shows significant anomalous concentration of $\mathrm{Sn}, \mathrm{W}, \mathrm{Ta}, \mathrm{Ti}$, and $\mathrm{Fe}$. $\mathrm{Au}, \mathrm{Cu}$ shows moderate to very high correlation. Areas of significant anomalous concentration were in the $2-4 \mathrm{~km}$ eastern outskirt of Kaiama and as well as 6-10 km south of Kaiama. Sn-W were more to the eastern outskirt while Sn-Ta were more in the southern part of Kaiama. All other trace elements V, Ni, Sr, Sb, Cd etc. shows low concentration with each other and are not significant in the mineralization of Kaiama. Further exploration work should be concentrated on soils and stream sediments towards delineating the possible ore deposit in the area of Kaiama and environs.

Keywords: Geostatistics; Geochemistry; Kaiama; Correlation matrix; Cluster analysis.

Copyright @ 2019: This is an open-access article distributed under the terms of the Creative Commons Attribution license which permits unrestricted use, distribution, and reproduction in any medium for non-commercial use (NonCommercial, or CC-BY-NC) provided the original author and source are credited.

\section{INTRODUCTION}

Kaiama northcentral Nigeria is becoming an area of mining interest due to the presence of various mineralization in the area. Artisanal mining activities have revealed the presence of wolframite, tantalite, cassitterite, copper, gold and various gem stones. However, a lot of unknown information still lingers about the mineralization in this region. This study aims to understand mineral association within three geologic media in the area of Kaiama namely; soil, stream sediments, and veins using geostatical tools on geochemical data from the area. This was done in order to bridge the knowledge gap about the mineralization and mineral association within the area of Kaiama,

Kaiama is the provincial capital of Kaiama Local Government Area in the northwestern part of Kwara State which itself is in the North-Central part of Nigeria. It lies within the Federal Survey map of
Nigeria Sheet 158 , Kaiama SE. The area is defined by Latitude N09 $300^{\circ} 00^{\prime \prime}$ to N09 $45^{\circ} 00^{\prime \prime}$ and Longitude $\mathrm{E} 003^{\circ} 45^{\circ} 00^{\prime \prime}$ to ${\mathrm{E} 004^{\circ}}^{\circ} 00^{\circ} 00^{\prime \prime}$ covering an approximate area extent of about $770 \mathrm{~km}^{2}$ (Fig-1). Kaiama is a border town, approximately $60 \mathrm{~km}$ from the international boundary between Nigeria and Benin Republic. Major towns within the area includes Kaiama, Kugeji, Kimenji, Bezira, Karo, Shirigberia, Banisula. Zekana, Gbetekuta, Kurogun. Kaiama is bounded by Niger state to the north, Oyo state to the south, and Baruten LGA of Kwara State to the west. The people speak Bokobaru mostly but have pockets of Fulani and Nupe indigenes.

Kaiama is readily accessible through the Ilorin-Kishi, the Jebba - Kainji road and KosubosuYashikira road (Fig-1). The roads are yet to be fully constructed. There are many untarred rural roads linking the major settlements. In addition, numerous 
footpaths links the settlement and farmlands. These footpaths aid accessibility to the outcrops in the study area. The roads in the area are better traversed during the dry season as flooding can make the town inaccessible during the raining season.

Kaiama falls within the south-western Basement Complex of Nigeria. The major lithological groups within the Basement Complex of Nigeria can be grouped into four different rock suits, namely; the
Migmatite Gneiss Complex (MGC), the Schist Belt (Meta-sedimentary and Meta-volcanic rocks), the Older Granites (Pan African granitoids), and the un-deformed Acid and Basic Dykes (Fig-2) [1]. These rocks constitute the Pre-Cambrian to Lower Palaeozoic Basement Complex rocks. The gneiss-migmatites bears imprints of the Liberian (ca. 2500Ma), Eburnean (ca. 2000Ma) and Pan African (ca. 600Ma) tectonic events $[2,3]$.

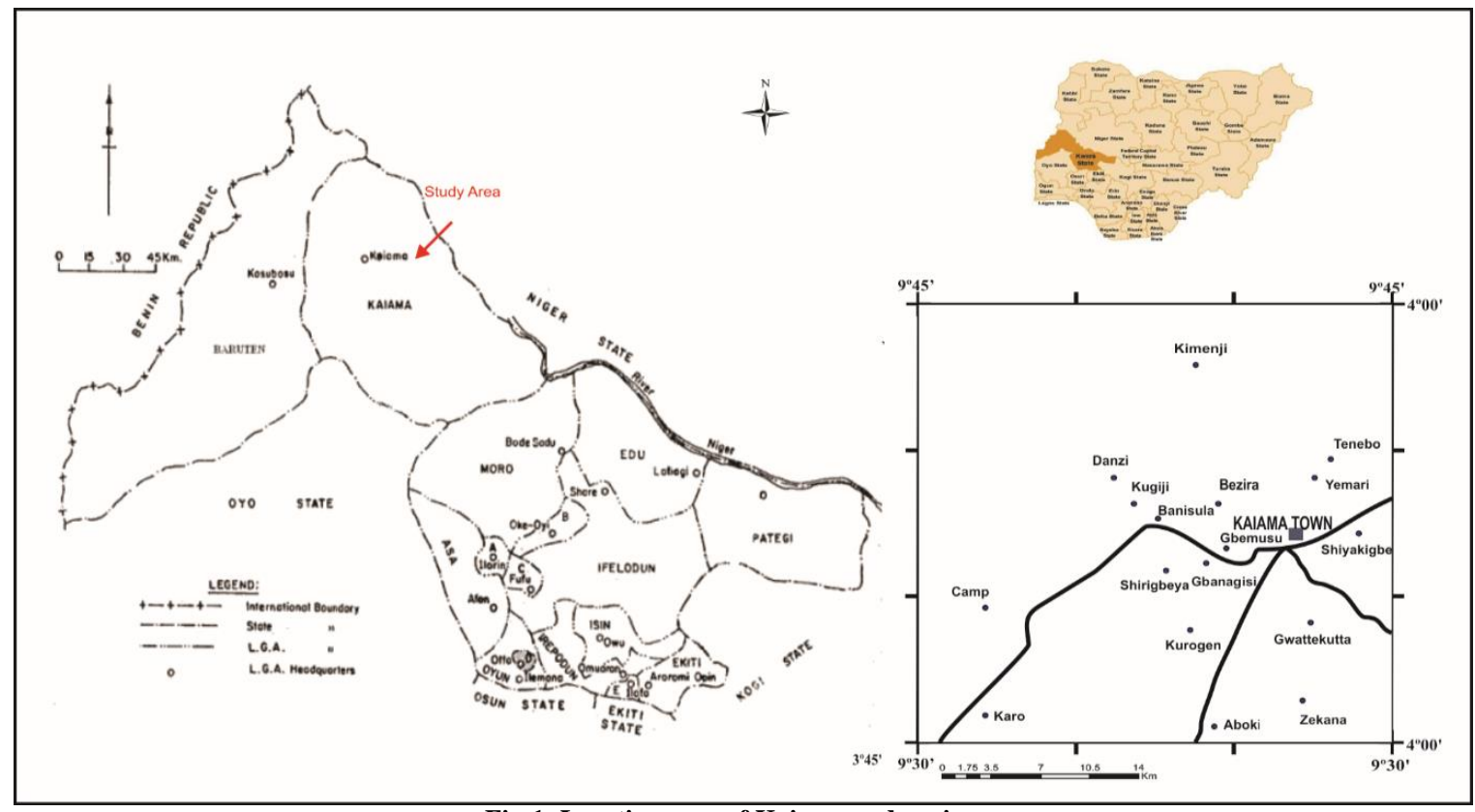

Fig-1: Location map of Kaiama and environs

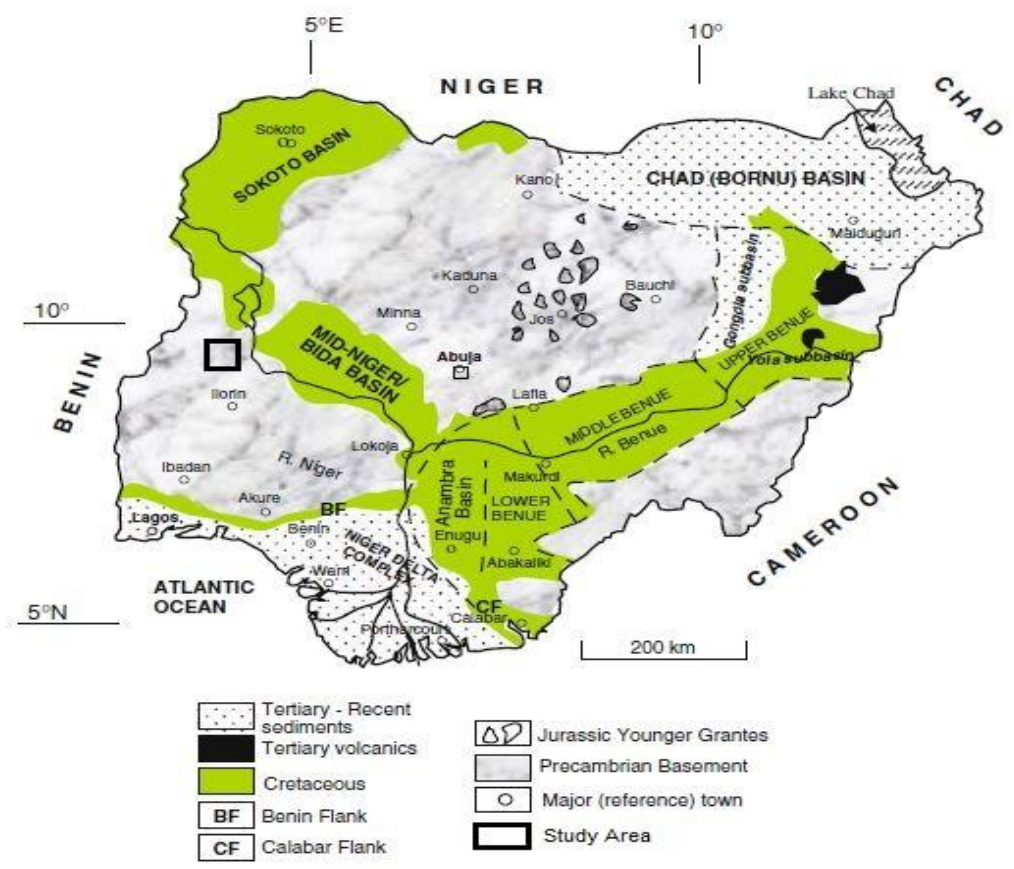

Fig-2: An overview of the geology of Nigeria showing the study area (Modified after Obaje 2009) [1]

Within the sequence are domains of metasediments and meta-volcanics intruded by igneous rocks, which constitute the north/south trending schist belts. The schist belt lithologies which consist of fine grained clastics, pelitic schists, phyllites, banded ironformations, marble and amphibolites are considered to 
be Upper Proterozoic assemblages [3]. They host most of the economic minerals in the Basement Complex. During the Pan-African episode, the Proterozoic migmatite-gneiss schist complex were intruded by various granitoids resulting from oceanic closure, subduction, oblique collision between the West African Craton and the Hoggar - Nigeria shields and crustal thickening [4].

The Nigerian basement complex is a polycyclic assemblage of heterogeneous migmatites and gneisses, metasediments and granites that have undergone a complex evolutionary history spanning through archaean to Pan-African (Late Proterozoic) times $[5,6]$. The rocks are believed to be the results of at least four major orogenic cycles of deformation, metamorphism and remobilization corresponding to the liberian $(2,650 \pm 150 \mathrm{Ma})$, the eburnean $(2,000 \pm 50$ $\mathrm{Ma})$, the kibaran $(1,100 \pm 200 \mathrm{Ma})$, and the Pan-African cycles (600 $\pm 150 \mathrm{Ma})$. Olade and Elueze [7], Ogezi [5] have favored largely ensialic processes in the evolution of the schist belts whilst Egbuniwe [8] stressed the importance of ensimatic processes in the formation of the schist belts.

The basement terrain is highly deformed as a result of various tectonic events with differing intensities. The resultant structure includes foliation (alternation of silicic-mafic minerals), minor folds, joints, fractures and faults. Each of the thermotectonic events produced characteristic imprints on the basement rocks. However, the Pan-African event was so pervasive that it nearly obliterated most of the structures of the earlier events, leaving behind mostly their traces [9-11]. Two major regional (transcurrent) fault systems (Anka-Yauri-Iseyin-AYI and Kalangai-ZungeruIfewara-KZI fault systems) trending NE-SW occur in Nigeria and these had probably developed during the late Pan-African orogeny [12]. These fault systems are dextral wrench faults with displacements in the order of tens of kilometers and have associated locally developed conjugate sinistral faults [13]. The most prominent structural trends are the N-S planar structures but relicts of E-W, NW-SE and NE-SW structures are preserved $[14,15]$.

The basement is also characterized by several sets of fractures with the north easterly and north westerly conjugate sets predominating [14]. ZungeruBirnin Gwari schist belt is a simple N-S syncline, 150 $\mathrm{km}$ long, with the northern part displaced dextrally by a NE-SW transcurrent fault. The Birnin Gwari schist belt occupies the synclinal axis of the belt and is characterized by conspicuous NNE-SSW trending ridges [16]. Kazaure schist belt is structurally simple, free from central granite intrusion and mafic igneous rock.

The Pan-African granites are usually elongated in a northern direction, an observation that shows that the Pan-African granite plutonism most probably exploited existing structures in the older basement [14]. Maru belt is a straight NNE-trending belt with a steeply dipping foliation [5]. Zuru schist belt can be described in terms of three phases of regional deformation D1, D2 and D3. D2, being the main cleavage-producing deformation phase that intensely folded the rocks into isoclinals N-S trending upright folds [17].

Locally, the various rock types within the study area include Granite, Schist, Migmatite, Quartz vein, Amphibolite, and Pegmatite vein. The pegmatite vein and the quartz vein are the main host of economic mineral signatures within the area of study of Kaiama.

\section{METHOD}

\section{Field Work and Geochemical Sampling}

Representative samples of rocks were collected from the field with the aid of block head hammer and chisel for petrographic studies. Using shovel and digger twenty-One pit at $2 \mathrm{~m}$ deep were dug at random but representative locations for soil samples and stream sediments along topographic lows and dried flood plains, cares was taken to exclude plant litters, roots and rock fragments [18, Salminen [19] randomly while some in the mining pit with the aid of digger and shovel to collect soil sediment and placed in a plastic self-seal bags for geochemical analyses. Locations of samples were taken and recorded while collected samples were carefully labeled and stored in polythene and cotton bags for further laboratory studies. Pictures of exposures were also taken using digital cameras.

Three field sampling pans were used to sample $20 \mathrm{~kg}$ stream sediments collected from first/second order streams (inner bent) from predetermined stream locations and hand dug pits were panned to recover heavy fractions that ore minerals are concentrated. The panning was carried out both on the field (streams) and Sedimentology Laboratory of Department of Geology, University Of Ilorin, Ilorin. The locations of the various samples collected are shown in Figure-3. The methodological process employed in the laboratory for the purpose of this study are; geochemical analysis for major, minor and trace elements and a petrographic study involving preparation and description of thin sections under petrological microscope. 


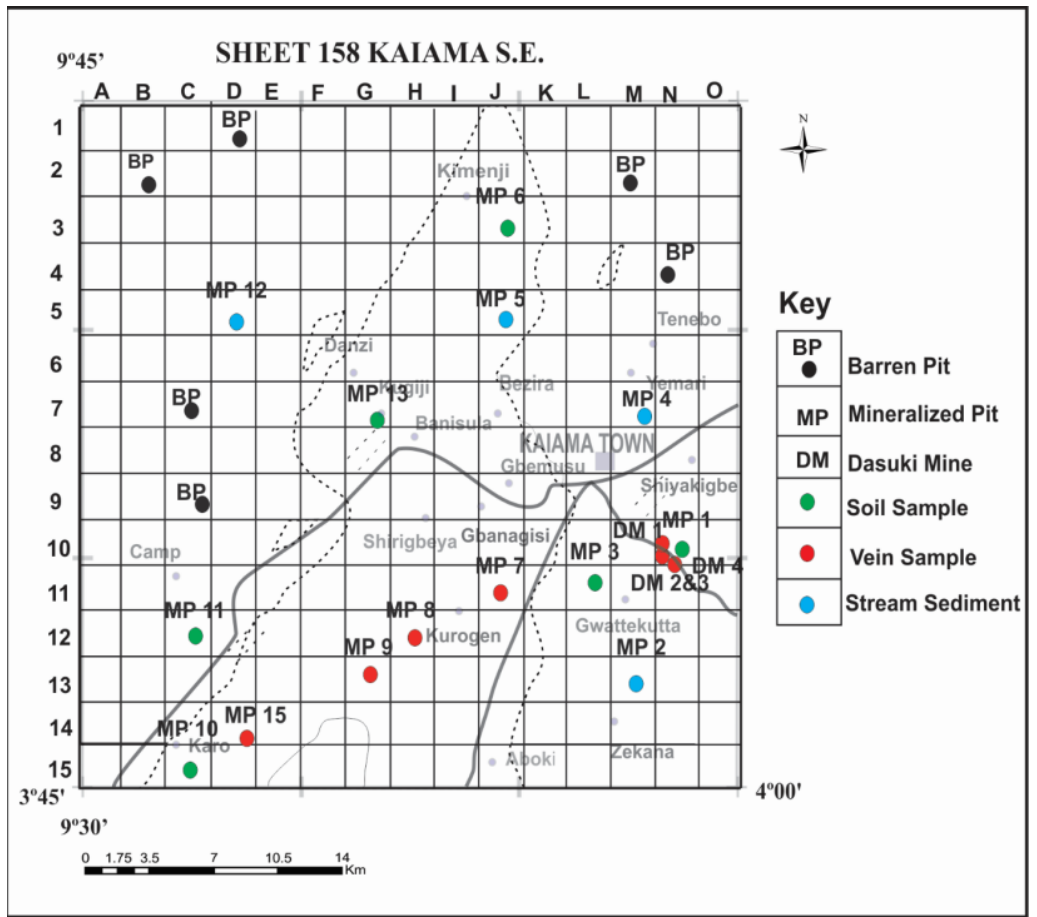

Fig-3: Location of sample collected for geochemical and petrological studies from Kaiama and environs

Selected rock were cut, crushed, quartered and pulverized for representative sample to be analyzed for geochemical composition, heavy fractions of soils and stream sediments obtained from panning were aired dried at room temperature after which they were also crushed, quartered and pulverized for major and trace geochemical composition analysis. The geochemical composition analysis was performed using are the X-ray Fluorescence Spectrometer (XRF) and Particle Induced $\mathrm{X}$-ray Emission Spectrometer (PIXE). The XRF was basically used to analyze for the major elements constituent while PIXE was used for trace elements contents. Analysis by XRF and PIXE was carried out at the laboratory of Energy Research Centre O.A.U Ile-Ife while complementary XRF was undertaken at the laboratory of Dangote Cement Ltd, Obajana kogi state.

\section{Statistical data analysis}

Multivariate statistical methods in the form of correlation matrix (CM), Factor analysis (FcA), Cluster Analysis (CA) classifications was performed to identify analogous behaviour among different parameters (metal contents and the physio-chemical characteristics of soil), and also among the samples along with hierarchical cluster analysis (HCA) were used to classify the elements into groups that may signify common origin. All multivariate statistical tests were performed using Statistical Program for Social Science SPSS (PASW Statistics 18) for Windows which represent a quantitative independent approach of samples and variables classification in environmental studies.

Analytical treatment of data was made mainly in Excel with parameters obtained include. Statistical analysis such as mean, standard deviation skewedness.
In addition, data from quartz veins, soil and stream sediment samples from geochemical analysis were treated using Pearson's correlation coefficient Allan et al., [20].

\section{RESULTS AND DISCUSSION Correlation Analysis}

In order to deduce the inter-relationships between the depths, elemental concentrations and physicochemical characteristics of the soils, cluster analysis and Pearson's correlation matrix was run on the experimental data between total elemental concentration in the samples and the clustering factors The results indicate that there is a generally strong to very strong positive correlation between total concentration and clustering of most of the elements that are highly correlated with one another represent geochemical associations, which indicate geologic or geochemical processes. Spearman correlation (rs) was used in order to assess the strength of the relationships between different elements [21], The Spearman correlation coefficient, rs, is significant in all cases reported in this section at 0.01 level of significance (that is, $\mathrm{p}=0.5)$.

\section{Trace Element Correlation}

The trace elements correlation values are given in Tables 1, 2, and 3 from quartz/pegmatite vein, soil, and stream sediments respectively.

In the quartz and pegmatite veins (Table-1) Ta and $\mathrm{Sn}$ shows high correlation $(\mathrm{r}=0.9219)$ as well as $\mathrm{Sn}$ and $\mathrm{Zn}(\mathrm{r}=0.8965), \mathrm{Pb}$ and $\mathrm{W}(\mathrm{r}=0.9999), \mathrm{Nb}$ and $\mathrm{Zn}$ $(\mathrm{r}=0.9810)$, and $\mathrm{Ti}$ and $\mathrm{Sr}(\mathrm{r}=0.8440)$. Other high correlation pair also exist but are not of significance to 
mineralization in Kaiama and environs. $\mathrm{V}$ and $\mathrm{S}$ shows moderate to strong correlation $(\mathrm{r}=0.5839)$ as well as $\mathrm{V}$ and $\mathrm{Ti}(\mathrm{r}=0.7409)$ while $\mathrm{S}$ and $\mathrm{Cr}(\mathrm{r}=-0.488), \mathrm{Pb}$ and $\mathrm{Sr}$ $(\mathrm{r}=-0.22875), \mathrm{Pb}$ and $\mathrm{Ti}(\mathrm{r}=-0.0637), \mathrm{Pb}$ and $\mathrm{Zn}(\mathrm{r}=-$ 0.29976) and $\mathrm{Pb}$ and $\mathrm{As}(\mathrm{r}=-0.45253)$ shows negative correlation.

From soil sample results (Table-2), $\mathrm{W}$ and $\mathrm{Zn}$ shows high correlation $(\mathrm{r}=0.8722)$ as well as $\mathrm{W}$ and $\mathrm{Ni}$ $(\mathrm{r}=0.7996), \mathrm{Ag}$ and $\mathrm{Cu}(\mathrm{r}=0.949), \mathrm{Au}$ and $\mathrm{Zn}(\mathrm{r}=0.868)$, and $\mathrm{Nb}$ and $\mathrm{Cu}(\mathrm{r}=0.9936)$, while $\mathrm{Ti}$ and $\mathrm{V}(\mathrm{r}=0.75531)$ shows moderate to strong correlation as well as $\mathrm{Cu}$ and $\mathrm{S} \quad(\mathrm{r}=0.727), \mathrm{Sn}$ and $\mathrm{Zn}(\mathrm{r}=0.5386), \mathrm{Sn}$ and $\mathrm{Cu}$ $(\mathrm{r}=0.667), \mathrm{Sn}$ and $\mathrm{W}(\mathrm{r}=0.5141), \mathrm{Sn}$ and $\mathrm{Au}(\mathrm{r}=0.5181)$, and $\mathrm{Sn}$ and $\mathrm{Ag}(\mathrm{r}=0.7233)$. Also, $\mathrm{S}$ and $\mathrm{Cr}(\mathrm{r}=-0.4907)$ shows negative to fair correlation as well $\mathrm{Sn}$ and $\mathrm{Ti}(\mathrm{r}=-$ $0.4355), \mathrm{W}$ and $\mathrm{S}$ ( $\mathrm{r}=-0.457), \mathrm{W}$ and $\mathrm{Ti}(\mathrm{r}=-0.2481)$, and $\mathrm{Nb}$ and $\mathrm{As}(\mathrm{r}=-0.2651)$.

From Table-3, Sn and V shows strong correlation $(r=0.8237)$, as well as $\mathrm{Sn}$ and $\mathrm{Ti}(\mathrm{r}=0.9412)$, $\mathrm{Au}$ and $\mathrm{Ni}(\mathrm{r}=0.8131)$, and $\mathrm{Au}$ and $\mathrm{W}(\mathrm{r}=0.9402)$, while $\mathrm{Sn}$ and Co shows moderate correlation $(\mathrm{r}=0.6553)$ as well as $\mathrm{Nb}$ and $\mathrm{Ag}(\mathrm{r}=0.45)$. Also, $\mathrm{Sn}$ and $\mathrm{W}$ shows negative correlation $(\mathrm{r}=-0.1915)$ as well as $\mathrm{Nb}$ and $\mathrm{Ti}$ $(\mathrm{r}=-0.2975)$, and $\mathrm{Sn}$ and $\mathrm{Nb}(\mathrm{r}=-0.5451)$.

Table-1: Correlation coefficients of trace element from Quartz/pegmatite vein in Kaiama and environs

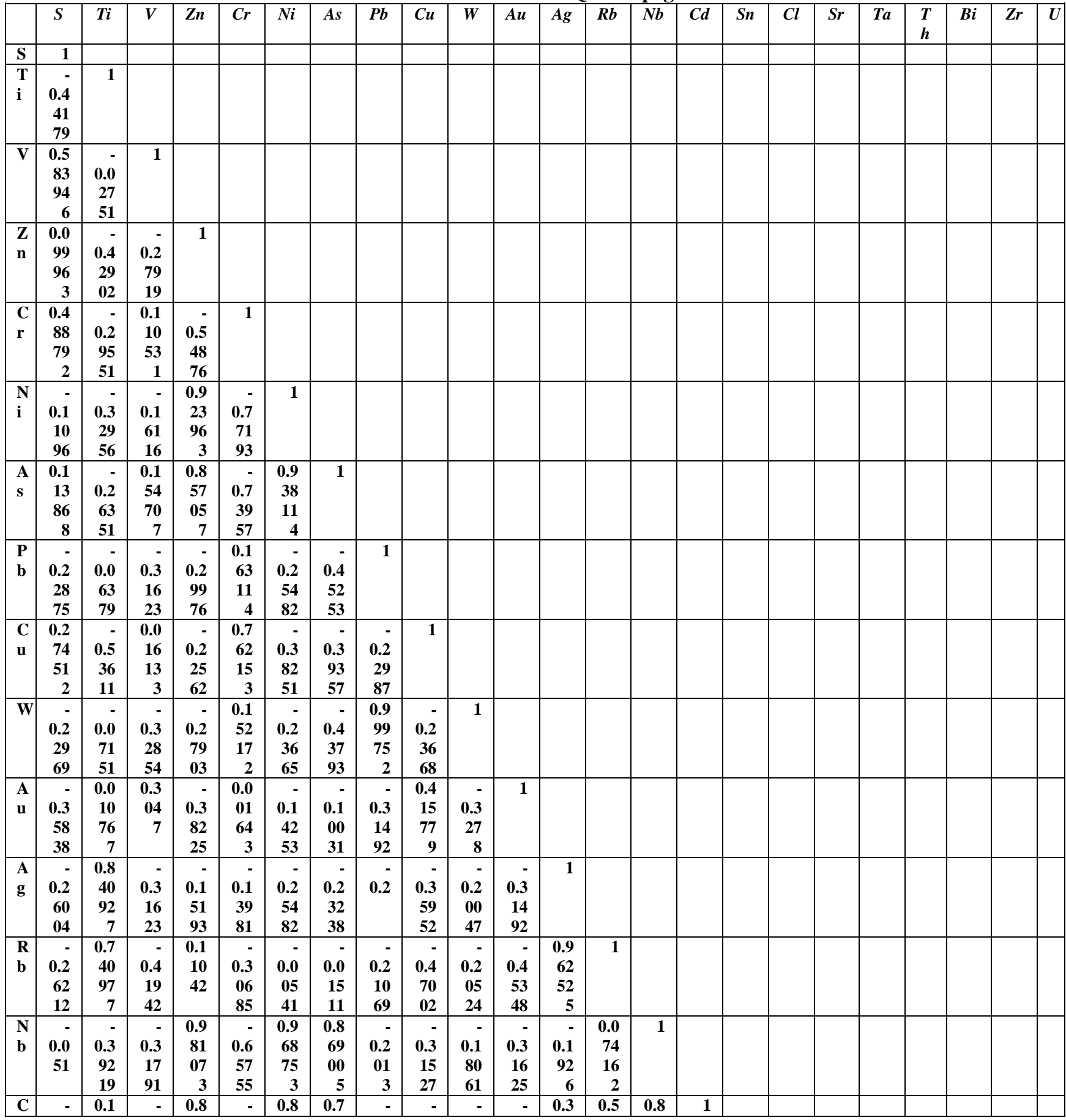




\begin{tabular}{|c|c|c|c|c|c|c|c|c|c|c|c|c|c|c|c|c|c|c|c|c|c|c|c|}
\hline d & $\begin{array}{r}0.2 \\
14 \\
41\end{array}$ & $\begin{array}{r}12 \\
38 \\
8\end{array}$ & $\begin{array}{r}0.4 \\
30 \\
04\end{array}$ & $\begin{array}{r}43 \\
15 \\
9\end{array}$ & $\begin{array}{r}0.7 \\
51 \\
61\end{array}$ & $\begin{array}{r}03 \\
60 \\
2\end{array}$ & $\begin{array}{r}32 \\
92 \\
8\end{array}$ & $\begin{array}{r}0.3 \\
25 \\
46\end{array}$ & $\begin{array}{r}0.5 \\
27 \\
49\end{array}$ & $\begin{array}{r}0.3 \\
06 \\
6\end{array}$ & $\begin{array}{r}0.4 \\
21 \\
98\end{array}$ & $\begin{array}{r}51 \\
86 \\
3\end{array}$ & $\begin{array}{r}82 \\
55 \\
6\end{array}$ & $\begin{array}{l}47 \\
32\end{array}$ & & & & & & & & & \\
\hline $\begin{array}{l}\text { S } \\
\text { n }\end{array}$ & $\begin{array}{r}- \\
0.1 \\
30 \\
72\end{array}$ & $\begin{array}{r}-\overline{0} \\
0.4 \\
22 \\
45\end{array}$ & $\begin{array}{r}- \\
0.4 \\
48 \\
42\end{array}$ & $\begin{array}{r}0.8 \\
67 \\
68 \\
6\end{array}$ & $\begin{array}{r} \\
0.5 \\
83 \\
56\end{array}$ & $\begin{array}{r}0.8 \\
65 \\
82 \\
4\end{array}$ & $\begin{array}{r}\mathbf{0 . 6} \\
87 \\
75 \\
7\end{array}$ & $\begin{array}{r}0.1 \\
91 \\
97 \\
7\end{array}$ & $\begin{array}{r} \\
0.4 \\
00 \\
06\end{array}$ & $\begin{array}{r}0.2 \\
12 \\
73 \\
1\end{array}$ & $\begin{array}{r} \\
0.4 \\
53 \\
61\end{array}$ & $\begin{array}{r}-\overline{0} \\
0.2 \\
66 \\
86\end{array}$ & $\begin{array}{r}-\overline{0} \\
0.0 \\
03 \\
25\end{array}$ & $\begin{array}{r}0.9 \\
22 \\
48 \\
9\end{array}$ & $\begin{array}{r}0.7 \\
21 \\
90 \\
4\end{array}$ & 1 & & & & & & & \\
\hline $\begin{array}{l}\text { C } \\
\text { l }\end{array}$ & $\begin{array}{r} \\
0.3 \\
04 \\
36\end{array}$ & $\begin{array}{r}-\overline{0} \\
0.3 \\
36 \\
25\end{array}$ & $\begin{array}{r} \\
0.1 \\
17 \\
18\end{array}$ & $\begin{array}{r}0.7 \\
44 \\
07 \\
7\end{array}$ & $\begin{array}{r}- \\
0.7 \\
88 \\
64\end{array}$ & $\begin{array}{r}0.9 \\
32 \\
93 \\
5\end{array}$ & $\begin{array}{r}0.8 \\
38 \\
44 \\
7\end{array}$ & $\begin{array}{r} \\
0.1 \\
03 \\
76\end{array}$ & $\begin{array}{r} \\
0.3 \\
52 \\
51\end{array}$ & $\begin{array}{r}- \\
0.0 \\
89 \\
61\end{array}$ & $\begin{array}{r}0.1 \\
19 \\
48 \\
1\end{array}$ & $\begin{array}{r}- \\
0.4 \\
29 \\
85\end{array}$ & $\begin{array}{r}- \\
0.2 \\
21 \\
19\end{array}$ & $\begin{array}{r}0.8 \\
44 \\
67 \\
5\end{array}$ & $\begin{array}{r}0.6 \\
05 \\
15 \\
3\end{array}$ & $\begin{array}{r}0.7 \\
95 \\
71 \\
6\end{array}$ & 1 & & & & & & \\
\hline $\begin{array}{l}\mathbf{S} \\
\mathbf{r}\end{array}$ & $\begin{array}{r}-\overline{0} \\
6.2 \\
04\end{array}$ & $\begin{array}{r}0.8 \\
40 \\
92 \\
7\end{array}$ & $\begin{array}{r}- \\
0.3 \\
16 \\
23\end{array}$ & $\begin{array}{r}- \\
0.1 \\
51 \\
93\end{array}$ & $\begin{array}{r}- \\
0.1 \\
39 \\
81\end{array}$ & $\begin{array}{r}- \\
0.2 \\
54 \\
82\end{array}$ & $\begin{array}{r}- \\
0.2 \\
32 \\
38\end{array}$ & $0 . \overline{2}$ & $\begin{array}{r}- \\
0.3 \\
59 \\
52\end{array}$ & $\begin{array}{r}- \\
0.2 \\
00 \\
47\end{array}$ & $\begin{array}{r}- \\
0.3 \\
14 \\
92\end{array}$ & 1 & $\begin{array}{r}0.9 \\
62 \\
52 \\
5\end{array}$ & $\begin{array}{r}- \\
0.1 \\
92 \\
6\end{array}$ & $\begin{array}{r}0.3 \\
51 \\
86 \\
3\end{array}$ & $\begin{array}{r}- \\
0.2 \\
66 \\
86\end{array}$ & $\begin{array}{r}- \\
0.4 \\
29 \\
85\end{array}$ & 1 & & & & & \\
\hline $\begin{array}{l}\mathbf{T} \\
\mathbf{a}\end{array}$ & $\begin{array}{r}- \\
0.0 \\
49 \\
86\end{array}$ & $\begin{array}{r}- \\
0.3 \\
91 \\
21\end{array}$ & $\begin{array}{r}- \\
0.3 \\
20 \\
66\end{array}$ & $\begin{array}{r}0.9 \\
81 \\
86 \\
9\end{array}$ & $\begin{array}{r}- \\
0.6 \\
55 \\
59\end{array}$ & $\begin{array}{r}0.9 \\
67 \\
54 \\
8\end{array}$ & $\begin{array}{r}\mathbf{0 . 8} \\
67 \\
63\end{array}$ & $\begin{array}{r}- \\
0.2 \\
02 \\
8\end{array}$ & $\begin{array}{r}- \\
0.3 \\
14 \\
02\end{array}$ & $\begin{array}{r}- \\
0.1 \\
82 \\
07\end{array}$ & $\begin{array}{r}- \\
0.3 \\
19 \\
33\end{array}$ & $\begin{array}{r}- \\
0.1 \\
88 \\
72\end{array}$ & $\begin{array}{r}0.0 \\
78 \\
13 \\
9\end{array}$ & $\begin{array}{r}0.9 \\
99 \\
98 \\
3\end{array}$ & $\begin{array}{r}0.8 \\
49 \\
06 \\
9\end{array}$ & $\begin{array}{r}0.9 \\
21 \\
97 \\
5\end{array}$ & $\begin{array}{r}0.8 \\
41 \\
75 \\
3\end{array}$ & $\begin{array}{r}0.1 \\
88 \\
72\end{array}$ & 1 & & & & \\
\hline $\begin{array}{l}\mathbf{T} \\
\mathbf{h}\end{array}$ & $\begin{array}{r}0.9 \\
87 \\
30 \\
3\end{array}$ & $\begin{array}{r}0.3 \\
42 \\
17\end{array}$ & $\begin{array}{r}0.6 \\
32 \\
45 \\
6\end{array}$ & $\begin{array}{r}- \\
0.0 \\
53 \\
38\end{array}$ & $\begin{array}{r}0.5 \\
59 \\
25\end{array}$ & $\begin{array}{r}- \\
0.2 \\
54 \\
82\end{array}$ & $\begin{array}{r}\mathbf{0 . 0} \\
12 \\
23\end{array}$ & 0.2 & $\begin{array}{r}0.2 \\
85 \\
09 \\
2\end{array}$ & $\begin{array}{r}- \\
0.2 \\
04 \\
13\end{array}$ & $\begin{array}{r}0.3 \\
14 \\
92\end{array}$ & 0.2 & $\begin{array}{r}- \\
0.2 \\
42 \\
53\end{array}$ & $\begin{array}{r}- \\
0.2 \\
03 \\
94\end{array}$ & $\begin{array}{r}- \\
0.3 \\
25 \\
46\end{array}$ & $\begin{array}{r}- \\
0.2 \\
72 \\
95\end{array}$ & $\begin{array}{r}- \\
0.4 \\
29 \\
85\end{array}$ & 0.2 & $\begin{array}{r}-\bar{r} \\
0.2 \\
02 \\
8\end{array}$ & 1 & & & \\
\hline $\begin{array}{l}\text { B } \\
\text { i }\end{array}$ & $\begin{array}{r}-\overline{0} \\
0.3 \\
29 \\
34\end{array}$ & $\begin{array}{r}0.3 \\
52 \\
74\end{array}$ & $\begin{array}{r}-\overline{0} \\
87 \\
62\end{array}$ & $\begin{array}{r}-\overline{0} \\
0.3 \\
49 \\
66\end{array}$ & $\begin{array}{r}0.5 \\
49 \\
61 \\
5\end{array}$ & $\begin{array}{r}- \\
0.3 \\
92 \\
93\end{array}$ & $\begin{array}{r}-\overline{ } \\
0.6 \\
30 \\
74\end{array}$ & $\begin{array}{r}0.4 \\
45 \\
46\end{array}$ & $\begin{array}{r}0.6 \\
57 \\
95\end{array}$ & $\begin{array}{r}0.4 \\
41 \\
29 \\
1\end{array}$ & $\begin{array}{r}0.3 \\
13 \\
25 \\
9\end{array}$ & $\begin{array}{r}- \\
0.3 \\
08 \\
4\end{array}$ & $\begin{array}{r} \\
0.3 \\
95 \\
52\end{array}$ & $\begin{array}{r}-\overline{0} \\
0.3 \\
12 \\
81\end{array}$ & $\begin{array}{r} \\
0.5 \\
01 \\
85\end{array}$ & $\begin{array}{r}0.1 \\
36 \\
08\end{array}$ & $\begin{array}{r}-\overline{0} \\
0.2 \\
14\end{array}$ & $\begin{array}{c}0.3 \\
08\end{array}$ & $\begin{array}{r}-\overline{0} \\
0.3 \\
12 \\
72\end{array}$ & $\begin{array}{r}- \\
0 . \\
30 \\
84\end{array}$ & 1 & & \\
\hline $\begin{array}{l}\mathbf{Z} \\
\mathbf{r}\end{array}$ & $\begin{array}{r}- \\
0.1 \\
00 \\
34\end{array}$ & $\begin{array}{r}0.2 \\
83 \\
87\end{array}$ & $\begin{array}{r}-\overline{0} \\
0.3 \\
16 \\
15\end{array}$ & $\begin{array}{r}0.9 \\
70 \\
78 \\
8\end{array}$ & $\begin{array}{r}- \\
0.7 \\
24 \\
32\end{array}$ & $\begin{array}{r}0.9 \\
72 \\
10 \\
1\end{array}$ & $\begin{array}{r}0.8 \\
84 \\
07 \\
8\end{array}$ & $\begin{array}{r}- \\
0.2 \\
47 \\
3\end{array}$ & $\begin{array}{r}- \\
0.3 \\
85\end{array}$ & $\begin{array}{r}- \\
0.2 \\
26 \\
9\end{array}$ & $\begin{array}{r}-\overline{0} \\
0.3 \\
09 \\
27\end{array}$ & $\begin{array}{r}-\overline{0} \\
\mathbf{9 7} \\
34\end{array}$ & $\begin{array}{r}0.1 \\
66 \\
42 \\
4\end{array}$ & $\begin{array}{r}0.9 \\
92 \\
87 \\
7\end{array}$ & $\begin{array}{r}0.8 \\
97 \\
24 \\
8\end{array}$ & $\begin{array}{r}0.8 \\
96 \\
54 \\
1\end{array}$ & $\begin{array}{r}0.8 \\
40 \\
94 \\
6\end{array}$ & $\begin{array}{r}\mathbf{0 . 0} \\
97 \\
34 \\
\end{array}$ & $\begin{array}{r}0.9 \\
92 \\
96 \\
7 \\
\end{array}$ & $\begin{array}{r}- \\
0 . \\
24 \\
73\end{array}$ & $\begin{array}{r}- \\
0.3 \\
81 \\
34\end{array}$ & 1 & \\
\hline $\mathbf{U}$ & $\begin{array}{r}- \\
0.0 \\
95 \\
9\end{array}$ & $\begin{array}{r}- \\
0 . \overline{52} \\
32\end{array}$ & $\begin{array}{r}-\overline{0.3} \\
81 \\
36\end{array}$ & $\begin{array}{r}0.9 \\
73 \\
29\end{array}$ & $\begin{array}{r}- \\
0.6 \\
91 \\
74\end{array}$ & $\begin{array}{r}0.9 \\
40 \\
84 \\
8\end{array}$ & $\begin{array}{r}0.8 \\
43 \\
01 \\
4\end{array}$ & $\begin{array}{r}-\overline{0} \\
0.2 \\
41 \\
2\end{array}$ & $\begin{array}{r}- \\
0.3 \\
82 \\
12\end{array}$ & $\begin{array}{r}-\overline{0} \\
0.2 \\
20 \\
17\end{array}$ & $\begin{array}{r}-\overline{0} \\
0.3 \\
79 \\
78\end{array}$ & $\begin{array}{r}- \\
0.0 \\
18 \\
55\end{array}$ & $\begin{array}{r}0.2 \\
46 \\
64 \\
1\end{array}$ & $\begin{array}{r}0.9 \\
84 \\
67 \\
2\end{array}$ & $\begin{array}{r}0.9 \\
25 \\
53 \\
7\end{array}$ & $\begin{array}{c}0.8 \\
92 \\
36\end{array}$ & $\begin{array}{r}0.7 \\
82 \\
39 \\
1\end{array}$ & $\begin{array}{r}0.0 \\
18 \\
55\end{array}$ & $\begin{array}{r}0.9 \\
85 \\
36 \\
3\end{array}$ & $\begin{array}{r}- \\
0 . \\
24 \\
12\end{array}$ & $\begin{array}{r}- \\
0.3 \\
71 \\
92\end{array}$ & $\begin{array}{r}0.9 \\
94 \\
06 \\
5\end{array}$ & 1 \\
\hline
\end{tabular}

Table-2: Correlation Coefficient of trace elements in Soil samples from Kaiama and Environs

\begin{tabular}{|c|c|c|c|c|c|c|c|c|c|c|c|c|c|c|c|c|c|c|c|c|c|}
\hline & $S$ & $T i$ & $V$ & $Z n$ & $C r$ & Co & $\mathrm{Ni}$ & $A s$ & $P b$ & $C u$ & $W$ & $A u$ & $A g$ & $R b$ & $N b$ & Mo & $C d$ & $S n$ & $S b$ & $\begin{array}{l}S \\
r\end{array}$ & $\begin{array}{l}\boldsymbol{H} \\
\boldsymbol{f}\end{array}$ \\
\hline $\mathbf{S}$ & 1 & & & & & & & & & & & & & & & & & & & & \\
\hline $\begin{array}{l}\text { T } \\
\text { i }\end{array}$ & $\begin{array}{r}-\overline{1} \\
0.1 \\
031 \\
6\end{array}$ & 1 & & & & & & & & & & & & & & & & & & & \\
\hline $\mathbf{V}$ & $\begin{array}{r}- \\
0.4 \\
667\end{array}$ & $\begin{array}{r}\mathbf{0 . 7} \\
\mathbf{5 3 1} \\
\mathbf{8 7}\end{array}$ & 1 & & & & & & & & & & & & & & & & & & \\
\hline $\begin{array}{l}\mathbf{Z} \\
\mathrm{n}\end{array}$ & $\begin{array}{r} \\
0.0 \\
514 \\
9\end{array}$ & $\begin{array}{r}-\bar{r} \\
0.3 \\
018 \\
5\end{array}$ & $\begin{array}{r}0.3 \\
713 \\
2\end{array}$ & 1 & & & & & & & & & & & & & & & & & \\
\hline $\begin{array}{l}\text { C } \\
\mathbf{r}\end{array}$ & $\begin{array}{r}0.4 \\
907 \\
92\end{array}$ & $\begin{array}{r} \\
0.3 \\
480 \\
8\end{array}$ & $\begin{array}{r}- \\
0.1 \\
226 \\
9\end{array}$ & $\begin{array}{r}0.2 \\
530 \\
48\end{array}$ & 1 & & & & & & & & & & & & & & & & \\
\hline $\begin{array}{l}\mathbf{C} \\
\mathbf{0}\end{array}$ & $\begin{array}{r}0.6 \\
592\end{array}$ & $\begin{array}{r}0.1 \\
938 \\
14\end{array}$ & $\begin{array}{r}0.6 \\
438 \\
02\end{array}$ & $\begin{array}{r}- \\
0.5 \\
422 \\
1\end{array}$ & $\begin{array}{r}- \\
0.1 \\
887 \\
7\end{array}$ & 1 & & & & & & & & & & & & & & & \\
\hline $\begin{array}{l}\mathbf{N} \\
\mathbf{i}\end{array}$ & $\begin{array}{r}- \\
0.0 \\
015 \\
5\end{array}$ & $\begin{array}{r}-\overline{0} \\
0.3 \\
948 \\
7\end{array}$ & $\begin{array}{r}- \\
0.4 \\
388 \\
3\end{array}$ & $\begin{array}{r}0.8 \\
626 \\
28\end{array}$ & $\begin{array}{r}0.1 \\
153 \\
31\end{array}$ & $\begin{array}{r}- \\
0.5 \\
014 \\
8\end{array}$ & 1 & & & & & & & & & & & & & & \\
\hline $\begin{array}{l}\mathbf{A} \\
\mathbf{s}\end{array}$ & $\begin{array}{r}-\bar{r} \\
0.4 \\
770 \\
6\end{array}$ & $\begin{array}{r}- \\
0.2 \\
336 \\
6\end{array}$ & $\begin{array}{r}- \\
0.2 \\
427 \\
7\end{array}$ & $\begin{array}{r}0.8 \\
526 \\
19\end{array}$ & $\begin{array}{r}- \\
0.2 \\
237 \\
9\end{array}$ & $\begin{array}{r}- \\
0.2 \\
749\end{array}$ & $\begin{array}{r}0.7 \\
842 \\
28\end{array}$ & 1 & & & & & & & & & & & & & \\
\hline $\begin{array}{l}\mathbf{P} \\
\text { b }\end{array}$ & $\begin{array}{r}0.2 \\
583 \\
96\end{array}$ & $\begin{array}{r}- \\
0.3 \\
495 \\
7\end{array}$ & $\begin{array}{r}- \\
0.0 \\
849\end{array}$ & $\begin{array}{r}0.5 \\
547 \\
97\end{array}$ & $\begin{array}{r}0.9 \\
243 \\
76\end{array}$ & $\begin{array}{r}- \\
0.2 \\
207 \\
9\end{array}$ & $\begin{array}{r}0.3 \\
973 \\
4\end{array}$ & $\begin{array}{r}0.1 \\
385 \\
5\end{array}$ & 1 & & & & & & & & & & & & \\
\hline $\begin{array}{l}\mathbf{C} \\
\mathbf{u}\end{array}$ & $\begin{array}{r}0.7 \\
274 \\
68\end{array}$ & $\begin{array}{r}-\overline{-} \\
0.3 \\
968\end{array}$ & $\begin{array}{r}- \\
0.4 \\
997\end{array}$ & $\begin{array}{r}0.1 \\
780 \\
27\end{array}$ & $\begin{array}{r}0.4 \\
877 \\
31\end{array}$ & $\begin{array}{r}-\overline{1} \\
830\end{array}$ & $\begin{array}{r}0.4 \\
800 \\
12\end{array}$ & $\begin{array}{r}- \\
0.1 \\
577\end{array}$ & $\begin{array}{r}0.4 \\
034 \\
6\end{array}$ & 1 & & & & & & & & & & & \\
\hline
\end{tabular}




\begin{tabular}{|c|c|c|c|c|c|c|c|c|c|c|c|c|c|c|c|c|c|c|c|c|c|c|}
\hline & & 5 & 8 & & & 4 & & 8 & & & & & & & & & & & & & & \\
\hline W & $\begin{array}{r}- \\
0.4 \\
572 \\
8\end{array}$ & $\begin{array}{r}- \\
0.2 \\
481 \\
3\end{array}$ & $\begin{array}{r}- \\
0.2 \\
490 \\
8\end{array}$ & $\begin{array}{r}0.8 \\
722 \\
01\end{array}$ & $\begin{array}{r}- \\
0.1 \\
821 \\
2\end{array}$ & $\begin{array}{r}- \\
0.2 \\
876 \\
7\end{array}$ & $\begin{array}{r}0.7 \\
996 \\
47\end{array}$ & $\begin{array}{r}0.9 \\
990 \\
64\end{array}$ & $\begin{array}{r}0.1 \\
806 \\
06\end{array}$ & $\begin{array}{r}- \\
0.1 \\
330 \\
9\end{array}$ & 1 & & & & & & & & & & & \\
\hline $\begin{array}{l}\mathbf{A} \\
\mathbf{u}\end{array}$ & $\begin{array}{r}- \\
0.4 \\
573\end{array}$ & $\begin{array}{r}-\overline{-} \\
0.2 \\
455 \\
6 \\
\end{array}$ & $\begin{array}{r}- \\
0.2 \\
513 \\
7 \\
\end{array}$ & $\begin{array}{r}0.8 \\
687 \\
23\end{array}$ & $\begin{array}{r} \\
0.1 \\
923 \\
9 \\
\end{array}$ & $\begin{array}{r} \\
0.2 \\
898\end{array}$ & $\begin{array}{r}0.7 \\
992 \\
73\end{array}$ & $\begin{array}{r}0.9 \\
994 \\
15\end{array}$ & $\begin{array}{r}0.1 \\
699 \\
73\end{array}$ & $\begin{array}{r} \\
0.1 \\
336 \\
3 \\
\end{array}$ & $\begin{array}{r}0.9 \\
999 \\
28\end{array}$ & 1 & & & & & & & & & & \\
\hline $\begin{array}{l}\mathbf{A} \\
\mathbf{g}\end{array}$ & $\begin{array}{r}0.6 \\
860 \\
93\end{array}$ & $\begin{array}{r}-\overline{0} \\
497 \\
6\end{array}$ & $\begin{array}{r}- \\
0.4 \\
611 \\
6\end{array}$ & $\begin{array}{r}0.0 \\
233 \\
11\end{array}$ & $\begin{array}{r}0.2 \\
125 \\
38\end{array}$ & $\begin{array}{r}- \\
0.4 \\
273 \\
1\end{array}$ & $\begin{array}{r}0.4 \\
055 \\
73\end{array}$ & $\begin{array}{r}- \\
0.2 \\
051 \\
2\end{array}$ & $\begin{array}{r}0.1 \\
093 \\
55\end{array}$ & $\begin{array}{r}0.9 \\
492 \\
98\end{array}$ & $\begin{array}{r}- \\
0.1 \\
921 \\
6\end{array}$ & $\begin{array}{r}- \\
0.1 \\
892 \\
9\end{array}$ & 1 & & & & & & & & & \\
\hline $\begin{array}{l}\mathbf{R} \\
\mathbf{b}\end{array}$ & $\begin{array}{r}0.5 \\
622 \\
35\end{array}$ & $\begin{array}{r}-\overline{2} \\
0.2 \\
844 \\
2\end{array}$ & $\begin{array}{r}- \\
0.1 \\
468 \\
5\end{array}$ & $\begin{array}{r}0.3 \\
265 \\
84\end{array}$ & $\begin{array}{r}0.9 \\
871 \\
69\end{array}$ & $\begin{array}{r} \\
0.3 \\
164\end{array}$ & $\begin{array}{r}0.1 \\
799 \\
9\end{array}$ & $\begin{array}{r} \\
0.1 \\
783 \\
8 \\
\end{array}$ & $\begin{array}{r}0.9 \\
274 \\
85\end{array}$ & $\begin{array}{r}0.5 \\
259 \\
86\end{array}$ & $\begin{array}{r}- \\
0.1 \\
361 \\
7\end{array}$ & $\begin{array}{r}- \\
0.1 \\
458 \\
5\end{array}$ & $\begin{array}{r}0.2 \\
576 \\
19\end{array}$ & 1 & & & & & & & & \\
\hline $\begin{array}{l}\mathbf{N} \\
\mathbf{b}\end{array}$ & $\begin{array}{r}0.7 \\
632 \\
64\end{array}$ & $\begin{array}{r}- \\
0.3 \\
427 \\
8\end{array}$ & $\begin{array}{r}- \\
0.4 \\
447 \\
3\end{array}$ & $\begin{array}{r}0.0 \\
793 \\
95\end{array}$ & $\begin{array}{r}0.4 \\
987 \\
01\end{array}$ & $\begin{array}{r}- \\
0.4 \\
429 \\
8\end{array}$ & $\begin{array}{r}0.3 \\
830 \\
04\end{array}$ & $\begin{array}{r}- \\
0.2 \\
656 \\
1\end{array}$ & $\begin{array}{r}0.3 \\
786 \\
54\end{array}$ & $\begin{array}{r}0.9 \\
936 \\
07\end{array}$ & $\begin{array}{r}- \\
0.2 \\
413 \\
8\end{array}$ & $\begin{array}{r}- \\
0.2 \\
419 \\
2\end{array}$ & $\begin{array}{r}0.9 \\
506 \\
84\end{array}$ & $\begin{array}{r}0.5 \\
331 \\
9\end{array}$ & 1 & & & & & & & \\
\hline $\begin{array}{l}\text { M } \\
\text { o }\end{array}$ & $\begin{array}{r}- \\
0.3 \\
429 \\
3\end{array}$ & $\begin{array}{r}0.7 \\
113 \\
21\end{array}$ & $\begin{array}{r}0.6 \\
892 \\
22\end{array}$ & $\begin{array}{r}0.0 \\
634 \\
74\end{array}$ & $\begin{array}{r}-\overline{0.1} \\
438\end{array}$ & $\begin{array}{r}0.4 \\
079 \\
02\end{array}$ & $\begin{array}{r}0.0 \\
491 \\
44\end{array}$ & $\begin{array}{r}0.1 \\
041 \\
43\end{array}$ & $\begin{array}{r}- \\
0.0 \\
005 \\
2\end{array}$ & $\begin{array}{r}- \\
0.1 \\
965 \\
3\end{array}$ & $\begin{array}{r}\mathbf{0 . 1} \\
024 \\
77\end{array}$ & $\begin{array}{r}0.1 \\
011 \\
33\end{array}$ & $\begin{array}{r}- \\
0.1 \\
183 \\
7\end{array}$ & $\begin{array}{r}- \\
0.1 \\
183 \\
2\end{array}$ & $\begin{array}{r}- \\
0.1 \\
891 \\
3\end{array}$ & 1 & & & & & & \\
\hline $\begin{array}{l}\text { C } \\
\text { d }\end{array}$ & $\begin{array}{r}0.8 \\
798 \\
48\end{array}$ & $\begin{array}{r}- \\
0.5 \\
275 \\
8\end{array}$ & $\begin{array}{r}- \\
0.6 \\
339 \\
3\end{array}$ & $\begin{array}{r}0.0 \\
836 \\
46\end{array}$ & $\begin{array}{r}0.6 \\
752 \\
69\end{array}$ & $\begin{array}{r}- \\
0.5 \\
999 \\
6\end{array}$ & $\begin{array}{r}0.1 \\
670 \\
39\end{array}$ & $\begin{array}{r}- \\
0.3 \\
360 \\
5\end{array}$ & $\begin{array}{r}0.4 \\
834 \\
54\end{array}$ & $\begin{array}{r}0.8 \\
111 \\
75\end{array}$ & $\begin{array}{r}- \\
0.3 \\
083 \\
9\end{array}$ & $\begin{array}{r}- \\
0.3 \\
109 \\
5\end{array}$ & $\begin{array}{r}0.6 \\
762 \\
62\end{array}$ & $\begin{array}{r}0.7 \\
006 \\
18\end{array}$ & $\begin{array}{r}0.8 \\
228 \\
99\end{array}$ & $\begin{array}{r}- \\
0.5 \\
895 \\
4\end{array}$ & 1 & & & & & \\
\hline $\begin{array}{l}\text { S } \\
\text { n }\end{array}$ & $\begin{array}{r}0.2 \\
116 \\
91\end{array}$ & $\begin{array}{r} \\
0.4 \\
355\end{array}$ & $\begin{array}{r}- \\
0.6 \\
011 \\
6\end{array}$ & $\begin{array}{r}0.5 \\
386 \\
43\end{array}$ & $\begin{array}{r}- \\
0.0 \\
685\end{array}$ & $\begin{array}{r}- \\
0.5 \\
205\end{array}$ & $\begin{array}{r}0.8 \\
669 \\
13\end{array}$ & $\begin{array}{r}0.5 \\
082 \\
8\end{array}$ & $\begin{array}{r}0.0 \\
833 \\
91\end{array}$ & $\begin{array}{r}0.6 \\
878 \\
55\end{array}$ & $\begin{array}{r}0.5 \\
141 \\
83\end{array}$ & $\begin{array}{r}0.5 \\
181 \\
03\end{array}$ & $\begin{array}{r}0.7 \\
233 \\
31\end{array}$ & $\begin{array}{r}- \\
0.0 \\
099\end{array}$ & $\begin{array}{r}0.6 \\
142 \\
29\end{array}$ & $\begin{array}{r}- \\
0.1 \\
147 \\
4\end{array}$ & $\begin{array}{r}0.3 \\
276 \\
52\end{array}$ & 1 & & & & \\
\hline $\begin{array}{l}\text { S } \\
\text { b }\end{array}$ & $\begin{array}{r}- \\
0.2 \\
410 \\
6\end{array}$ & $\begin{array}{r}0.4 \\
682 \\
03\end{array}$ & $\begin{array}{r}0.6 \\
436 \\
79\end{array}$ & $\begin{array}{r}- \\
0.0 \\
005 \\
6\end{array}$ & $\begin{array}{r}- \\
0.1 \\
204 \\
7\end{array}$ & $\begin{array}{r}\mathbf{0 . 1} \\
133 \\
77\end{array}$ & $\begin{array}{r}0.1 \\
850 \\
48\end{array}$ & $\begin{array}{r}0.0 \\
871 \\
59\end{array}$ & $\begin{array}{r}0.0 \\
288 \\
74\end{array}$ & $\begin{array}{r}0.0 \\
280 \\
86\end{array}$ & $\begin{array}{r}0.0 \\
876 \\
54\end{array}$ & $\begin{array}{r}0.0 \\
883 \\
6\end{array}$ & $\begin{array}{r}\mathbf{0 . 0} \\
595 \\
52\end{array}$ & $\begin{array}{r}- \\
0.0 \\
823\end{array}$ & $\begin{array}{r}0.0 \\
390 \\
16\end{array}$ & $\begin{array}{r}0.4 \\
374 \\
94\end{array}$ & $\begin{array}{r}- \\
0.2 \\
993 \\
2\end{array}$ & $\begin{array}{r}0.1 \\
050 \\
43\end{array}$ & 1 & & & \\
\hline $\begin{array}{l}S \\
\mathbf{r}\end{array}$ & $\begin{array}{r}0.2 \\
128 \\
67\end{array}$ & $\begin{array}{r}- \\
0.2 \\
918 \\
1\end{array}$ & $\begin{array}{r}- \\
0.4 \\
610 \\
4\end{array}$ & $\begin{array}{r}- \\
0.2 \\
050 \\
6\end{array}$ & $\begin{array}{r}- \\
0.1 \\
098 \\
2\end{array}$ & $\begin{array}{r}-\bar{r} \\
0.3 \\
063 \\
4\end{array}$ & $\begin{array}{r}-\bar{r} \\
0.4 \\
102 \\
7\end{array}$ & $\begin{array}{r}- \\
0.1 \\
666 \\
7\end{array}$ & $\begin{array}{r}- \\
0.2 \\
760 \\
4\end{array}$ & $\begin{array}{r}- \\
0.2 \\
825 \\
6\end{array}$ & $\begin{array}{r}- \\
0.1 \\
784 \\
6\end{array}$ & $\begin{array}{r}- \\
0.1 \\
758 \\
6\end{array}$ & $\begin{array}{r}- \\
0.2 \\
948 \\
6\end{array}$ & $\begin{array}{r}- \\
0.1 \\
174 \\
1\end{array}$ & $\begin{array}{r}- \\
0.2 \\
7\end{array}$ & $\begin{array}{r}- \\
0.7 \\
988 \\
3\end{array}$ & $\begin{array}{r}0.2 \\
454 \\
88\end{array}$ & $\begin{array}{r}- \\
0.3 \\
084 \\
7\end{array}$ & $\begin{array}{r}-\overline{0} \\
0.5 \\
91 \\
06\end{array}$ & 1 & & \\
\hline $\begin{array}{l}\mathbf{H} \\
\mathbf{f}\end{array}$ & $\begin{array}{r}0.2 \\
128 \\
67\end{array}$ & $\begin{array}{r}- \\
0.2 \\
918 \\
1\end{array}$ & $\begin{array}{r}- \\
0.4 \\
610 \\
4\end{array}$ & $\begin{array}{r}- \\
0.2 \\
050 \\
6\end{array}$ & $\begin{array}{r}- \\
0.1 \\
098 \\
2\end{array}$ & $\begin{array}{r}- \\
0.3 \\
063 \\
4\end{array}$ & $\begin{array}{r}- \\
0.4 \\
102 \\
7\end{array}$ & $\begin{array}{r}- \\
0.1 \\
666 \\
7\end{array}$ & $\begin{array}{r}-\bar{r} \\
0.2 \\
760 \\
4\end{array}$ & $\begin{array}{r}- \\
0.2 \\
825 \\
6\end{array}$ & $\begin{array}{r}- \\
0.1 \\
784 \\
6\end{array}$ & $\begin{array}{r}- \\
0.1 \\
758 \\
6\end{array}$ & $\begin{array}{r}- \\
0.2 \\
948 \\
6\end{array}$ & $\begin{array}{r}- \\
0.1 \\
174 \\
1\end{array}$ & $\begin{array}{r}- \\
0.2 \\
7\end{array}$ & $\begin{array}{r}- \\
0.7 \\
988 \\
3\end{array}$ & $\begin{array}{r}0.2 \\
454 \\
88\end{array}$ & $\begin{array}{r}- \\
0.3 \\
084 \\
7\end{array}$ & $\begin{array}{r}- \\
0.5 \\
91 \\
06\end{array}$ & 1 & 1 & \\
\hline $\begin{array}{l}\mathbf{Z} \\
\mathbf{r}\end{array}$ & $\begin{array}{r}0.2 \\
128 \\
67\end{array}$ & $\begin{array}{r}- \\
0.2 \\
918 \\
1\end{array}$ & $\begin{array}{r}- \\
0.4 \\
610 \\
4\end{array}$ & $\begin{array}{r}- \\
0.2 \\
050 \\
6\end{array}$ & $\begin{array}{r}- \\
0.1 \\
098 \\
2\end{array}$ & $\begin{array}{r}- \\
0.3 \\
063 \\
4\end{array}$ & $\begin{array}{r}- \\
0.4 \\
102 \\
7\end{array}$ & $\begin{array}{r}- \\
0.1 \\
666 \\
7\end{array}$ & $\begin{array}{r}- \\
0.2 \\
760 \\
4\end{array}$ & $\begin{array}{r}- \\
0.2 \\
825 \\
6\end{array}$ & $\begin{array}{r}- \\
0.1 \\
784 \\
6\end{array}$ & $\begin{array}{r}- \\
0.1 \\
758 \\
6\end{array}$ & $\begin{array}{r}- \\
0.2 \\
948 \\
6\end{array}$ & $\begin{array}{r}- \\
0.1 \\
174 \\
1\end{array}$ & $\begin{array}{r}- \\
0.2 \\
7\end{array}$ & $\begin{array}{r}- \\
0.7 \\
988 \\
3\end{array}$ & $\begin{array}{r}0.2 \\
454 \\
88\end{array}$ & $\begin{array}{r}- \\
0.3 \\
084 \\
7\end{array}$ & $\begin{array}{r}- \\
0.5 \\
91 \\
06\end{array}$ & 1 & 1 & 1 \\
\hline
\end{tabular}

Table 3: Correlation Coefficient of trace elements in Soil samples from Kaiama and Environs

\begin{tabular}{|c|c|c|c|c|c|c|c|c|c|c|c|c|c|c|c|c|}
\hline & $S$ & $T i$ & $\boldsymbol{V}$ & $Z n$ & $\mathrm{Cr}$ & Co & $\mathrm{Ni}$ & $P b$ & $C u$ & $W$ & $A u$ & $A g$ & $R \boldsymbol{R}$ & $N b$ & Mo & $S n$ \\
\hline $\mathbf{S}$ & 1 & & & & & & & & & & & & & & & \\
\hline $\begin{array}{l}\text { T } \\
\mathbf{i}\end{array}$ & $\begin{array}{r}- \\
0.141 \\
26\end{array}$ & 1 & & & & & & & & & & & & & & \\
\hline $\mathbf{V}$ & $\begin{array}{r}- \\
0.052 \\
44\end{array}$ & $\begin{array}{r}0.899 \\
428\end{array}$ & 1 & & & & & & & & & & & & & \\
\hline $\begin{array}{l}\mathbf{Z} \\
\mathrm{n}\end{array}$ & $\begin{array}{r}\mathbf{0 . 5 5 6} \\
887\end{array}$ & $\begin{array}{r}- \\
0.722 \\
49\end{array}$ & $\begin{array}{r}- \\
0.582 \\
75\end{array}$ & 1 & & & & & & & & & & & & \\
\hline $\begin{array}{l}\text { C } \\
\text { r }\end{array}$ & $\begin{array}{r}- \\
0.662 \\
12 \\
\end{array}$ & 0.281 & $\begin{array}{r}- \\
0.516 \\
89 \\
\end{array}$ & $\begin{array}{r}0.056 \\
614\end{array}$ & 1 & & & & & & & & & & & \\
\hline $\begin{array}{l}\text { C } \\
\text { o }\end{array}$ & $\begin{array}{r}- \\
0.445 \\
71\end{array}$ & $\begin{array}{r}0.580 \\
796\end{array}$ & $\begin{array}{r}\mathbf{0 . 8 0 7} \\
45\end{array}$ & $\begin{array}{r}- \\
0.533 \\
24\end{array}$ & $\begin{array}{r}- \\
0.258 \\
02\end{array}$ & 1 & & & & & & & & & & \\
\hline $\begin{array}{l}\mathbf{N} \\
\mathbf{i}\end{array}$ & $\begin{array}{r}0.482 \\
085\end{array}$ & $\begin{array}{r}- \\
0.634 \\
29\end{array}$ & $\begin{array}{r}-\overline{0.463} \\
33\end{array}$ & $\begin{array}{r}\mathbf{0 . 9 8 0} \\
534\end{array}$ & $\begin{array}{r}0.079 \\
57\end{array}$ & $\begin{array}{r}-\overline{0} \\
0.383 \\
12\end{array}$ & 1 & & & & & & & & & \\
\hline $\begin{array}{l}\mathbf{P} \\
\text { b }\end{array}$ & 0.662 & 0.281 & 0.516 & $\begin{array}{r}0.056 \\
614\end{array}$ & 1 & $\begin{array}{r}- \\
0.258\end{array}$ & $\begin{array}{r}0.079 \\
57\end{array}$ & 1 & & & & & & & & \\
\hline
\end{tabular}




\begin{tabular}{|c|c|c|c|c|c|c|c|c|c|c|c|c|c|c|c|c|c|}
\hline & 12 & & 89 & & & 02 & & & & & & & & & & & \\
\hline $\begin{array}{l}\mathbf{C} \\
\mathbf{u}\end{array}$ & $\begin{array}{r}0.812 \\
4\end{array}$ & $\begin{array}{r}- \\
0.249 \\
75\end{array}$ & $\begin{array}{r}- \\
0.409 \\
05\end{array}$ & $\begin{array}{r}0.480 \\
019\end{array}$ & $\begin{array}{r}- \\
0.224 \\
65\end{array}$ & $\begin{array}{r}- \\
0.844 \\
46\end{array}$ & $\begin{array}{r}0.345 \\
023\end{array}$ & $\begin{array}{r}- \\
0.224 \\
65\end{array}$ & 1 & & & & & & & & \\
\hline $\mathbf{W}$ & $\begin{array}{r}0.169 \\
419\end{array}$ & $\begin{array}{r}- \\
0.231 \\
07 \\
\end{array}$ & $\begin{array}{r}0.174 \\
288\end{array}$ & $\begin{array}{r}\mathbf{0 . 5 1 3} \\
597\end{array}$ & $\begin{array}{r}- \\
0.275 \\
12 \\
\end{array}$ & $\begin{array}{r}0.442 \\
583\end{array}$ & $\begin{array}{r}0.628 \\
982\end{array}$ & $\begin{array}{r}- \\
0.275 \\
12 \\
\end{array}$ & $\begin{array}{r}- \\
0.337 \\
71 \\
\end{array}$ & 1 & & & & & & & \\
\hline $\begin{array}{l}\mathbf{A} \\
\mathbf{u}\end{array}$ & $\begin{array}{r}0.456 \\
115\end{array}$ & $\begin{array}{r}- \\
0.365 \\
91\end{array}$ & $\begin{array}{r}0.002 \\
407\end{array}$ & $\begin{array}{r}0.739 \\
651\end{array}$ & $\begin{array}{r}- \\
0.334 \\
26\end{array}$ & $\begin{array}{r}0.145 \\
207\end{array}$ & $\begin{array}{r}0.813 \\
161\end{array}$ & $\begin{array}{r}- \\
0.334 \\
26\end{array}$ & $\begin{array}{r}0.000 \\
288\end{array}$ & $\begin{array}{r}0.940 \\
227\end{array}$ & 1 & & & & & & \\
\hline $\begin{array}{l}\mathbf{A} \\
\mathbf{g}\end{array}$ & $\begin{array}{r}0.113 \\
178\end{array}$ & $\begin{array}{r}- \\
0.388 \\
97 \\
\end{array}$ & $\begin{array}{r} \\
0.568 \\
5\end{array}$ & $\begin{array}{r}- \\
0.119 \\
17\end{array}$ & 0 & $\begin{array}{r}- \\
0.624 \\
53\end{array}$ & $\begin{array}{r}- \\
0.308 \\
23\end{array}$ & $\begin{array}{r} \\
2.345 \\
7\end{array}$ & $\begin{array}{r}0.463 \\
872\end{array}$ & $\begin{array}{r}- \\
0.673 \\
42\end{array}$ & $\begin{array}{r}- \\
0.544 \\
68\end{array}$ & 1 & & & & & \\
\hline $\begin{array}{l}\mathbf{R} \\
\mathbf{b}\end{array}$ & $\begin{array}{r} \\
0.662 \\
12\end{array}$ & 0.281 & $\begin{array}{r} \\
0.516 \\
89\end{array}$ & $\begin{array}{r}0.056 \\
614\end{array}$ & 1 & $\begin{array}{r}- \\
0.258 \\
02\end{array}$ & $\begin{array}{r}0.079 \\
57\end{array}$ & 1 & $\begin{array}{r}- \\
0.224 \\
65 \\
\end{array}$ & $\begin{array}{r}- \\
0.275 \\
12\end{array}$ & $\begin{array}{r}- \\
0.334 \\
26\end{array}$ & 0 & 1 & & & & \\
\hline $\begin{array}{l}\mathbf{N} \\
\mathbf{b}\end{array}$ & $\begin{array}{r}0.882 \\
127\end{array}$ & $\begin{array}{r}- \\
0.297 \\
52\end{array}$ & $\begin{array}{r}- \\
0.388 \\
67 \\
\end{array}$ & $\begin{array}{r}\mathbf{0 . 5 3 0} \\
851\end{array}$ & $\begin{array}{r} \\
0.342 \\
05\end{array}$ & $\begin{array}{r}- \\
0.796 \\
82 \\
\end{array}$ & $\begin{array}{r}0.396 \\
006\end{array}$ & $\begin{array}{r}- \\
0.342 \\
05 \\
\end{array}$ & $\begin{array}{r}0.986 \\
562\end{array}$ & $\begin{array}{r}- \\
0.219 \\
81 \\
\end{array}$ & $\begin{array}{r}0.117 \\
919\end{array}$ & $\begin{array}{r}0.450 \\
145\end{array}$ & $\begin{array}{r} \\
0.342 \\
05\end{array}$ & 1 & & & \\
\hline $\begin{array}{l}\mathbf{M} \\
\mathbf{0}\end{array}$ & $\begin{array}{r}- \\
0.170 \\
29\end{array}$ & $\begin{array}{r}- \\
0.332 \\
68 \\
\end{array}$ & $\begin{array}{r}0.049 \\
201\end{array}$ & $\begin{array}{r}\mathbf{0 . 0 2 4} \\
511\end{array}$ & $\begin{array}{r}- \\
0.313 \\
89\end{array}$ & $\begin{array}{r}0.502 \\
971\end{array}$ & $\begin{array}{r}\mathbf{0 . 0 5 2} \\
535\end{array}$ & $\begin{array}{r}- \\
0.313 \\
89 \\
\end{array}$ & $\begin{array}{r}- \\
0.556 \\
22\end{array}$ & $\begin{array}{r}0.629 \\
116\end{array}$ & $\begin{array}{r}0.463 \\
328\end{array}$ & $\begin{array}{r}- \\
0.056 \\
96\end{array}$ & $\begin{array}{r}- \\
0.313 \\
89\end{array}$ & $\begin{array}{r}- \\
0.42 \\
315\end{array}$ & 1 & & \\
\hline $\begin{array}{l}\text { S } \\
\text { n }\end{array}$ & $\begin{array}{r}- \\
0.427 \\
57\end{array}$ & $\begin{array}{r}0.941 \\
283\end{array}$ & $\begin{array}{r}0.823 \\
725\end{array}$ & $\begin{array}{r}- \\
0.735 \\
37\end{array}$ & $\begin{array}{r}0.014 \\
437\end{array}$ & $\begin{array}{r}0.655 \\
355\end{array}$ & $\begin{array}{r}- \\
0.613 \\
34 \\
\end{array}$ & $\begin{array}{r}0.014 \\
437\end{array}$ & $\begin{array}{r}- \\
0.473 \\
83 \\
\end{array}$ & $\begin{array}{r}- \\
0.191 \\
51\end{array}$ & $\begin{array}{r}- \\
0.393 \\
45 \\
\end{array}$ & $\begin{array}{r}- \\
0.504 \\
18\end{array}$ & $\begin{array}{r}0.014 \\
437\end{array}$ & $\begin{array}{r}- \\
0.54 \\
51\end{array}$ & $\begin{array}{r}- \\
0.30 \\
455 \\
\end{array}$ & 1 & \\
\hline $\begin{array}{l}\mathbf{S} \\
\mathbf{b}\end{array}$ & $\begin{array}{r}- \\
0.773 \\
58 \\
\end{array}$ & $\begin{array}{r}- \\
0.285 \\
6\end{array}$ & $\begin{array}{r}- \\
0.454 \\
34\end{array}$ & $\begin{array}{r}- \\
0.001 \\
55\end{array}$ & $\begin{array}{r}0.977 \\
334\end{array}$ & $\begin{array}{r}- \\
0.092 \\
2\end{array}$ & $\begin{array}{r}0.040 \\
67\end{array}$ & $\begin{array}{r}0.977 \\
334\end{array}$ & $\begin{array}{r}- \\
0.409 \\
65\end{array}$ & $\begin{array}{r}- \\
0.157 \\
74\end{array}$ & $\begin{array}{r}- \\
0.277 \\
14\end{array}$ & $\begin{array}{r}- \\
0.061 \\
78 \\
\end{array}$ & $\begin{array}{r}0.977 \\
334\end{array}$ & $\begin{array}{r}- \\
0.50 \\
827 \\
\end{array}$ & $\begin{array}{r}- \\
0.12 \\
389\end{array}$ & $\begin{array}{r}0.036 \\
559\end{array}$ & 1 \\
\hline
\end{tabular}

\section{Cluster Analysis}

This was based on the high concentrations of some elements obtained in certain sampling sites and the strong correlation between the elements indicated by the Spearman correlation matrix.

Cluster analysis (CA) is a multivariate procedure for detecting natural grouping in data. Cluster analysis classification is based on placing objects into more or less homogenous groups to reveal relationship between the groups. The results of the geochemical analysis were subjected to multivariate analysis (CA) using SPSS software programme of Department of statistic, University of Ilorin, Nigeria. The elements were grouped into factors based on their loading in each factor (i.e. they are placed in a factor group where they have heavy loading).

\section{Dendogram}

Dendogram is a hierarchic classification represented by a two-dimensional diagram that illustrates the fusions or divisions made at each successive stage of the analysis [22]. The dendograms of the cluster analysis of both the major oxides and trace elements are shown in Fig 4-9 which allowed the grouping of homogenous clusters groups.

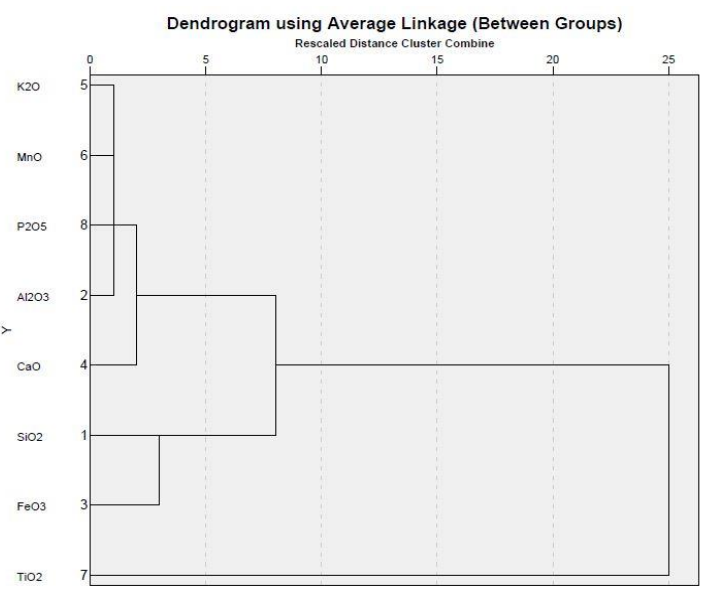

Fig-4: Dendogram image showing clusters of homogenous grouping of Major Oxides in Quartz/pegmatite Vein of Kaiama and environs

In the quartz and pegmatite veins of Kaiama and environs, five major factors with 7 stages/cases were involved. The first cluster has $\mathrm{K}_{2} \mathrm{O}, \mathrm{MnO}, \mathrm{P}_{2} \mathrm{O}_{5}$, and $\mathrm{Al}_{2} \mathrm{O}_{3}$ while the second stage involved $\mathrm{P}_{2} \mathrm{O}_{5}, \mathrm{Al}_{2} \mathrm{O}_{3}$ and $\mathrm{CaO}$. The third stage had $\mathrm{Al}_{2} \mathrm{O}_{3}, \mathrm{CaO}$, and $\mathrm{SiO}_{2}$
(Albititization) while the fourth stage were $\mathrm{SiO}_{2}, \mathrm{FeO} 3$. The fifth cluster had $\mathrm{CaO}, \mathrm{SiO}_{2}, \mathrm{FeO}_{3}$, and $\mathrm{TiO}$ 2. The first cluster/factor further strengthen the findings from the Spearman correlation while the fifth stage underlined the aluminosilicate composition. 


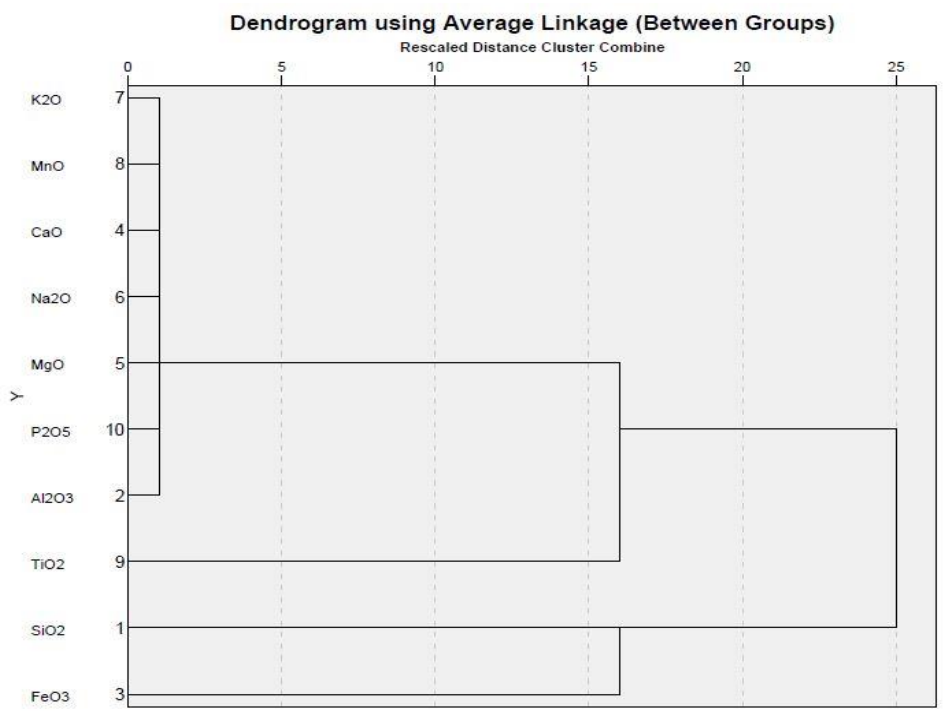

Fig-5: Dendogram image showing clusters of mineral assemblages of Major Oxides in Soils of Kaiama and environs

The soil samples analysis comprised of 9 stages with 4 clusters, the first stage is $\mathrm{K}_{2} \mathrm{O}-\mathrm{MnO}-$ $\mathrm{CaO}-\mathrm{Na}_{2} \mathrm{O}, \mathrm{MgO}, \mathrm{P}_{2} \mathrm{O}_{5}, \mathrm{Al}_{2} \mathrm{O}_{3}$ and can be said to be supergene/mineralization related alteration suite based on their association with clays and wall rock alteration association. The second cluster $\mathrm{MgO}, \mathrm{P}_{2} \mathrm{O}_{5}, \mathrm{Al}_{2} \mathrm{O}_{3}$, and TiO2. The third stage had $\mathrm{P}_{2} \mathrm{O}_{5}, \mathrm{Al}_{2} \mathrm{O}_{3}, \mathrm{Tio}_{2}$, and $\mathrm{SiO}_{2}$ with the highest loading while the $4^{\text {th }}$ stage was $\mathrm{SiO}_{2}$. $\mathrm{FeO} 3$. The $2^{\text {nd }}$ and $4^{\text {th }}$ stages were significantly iron rich.

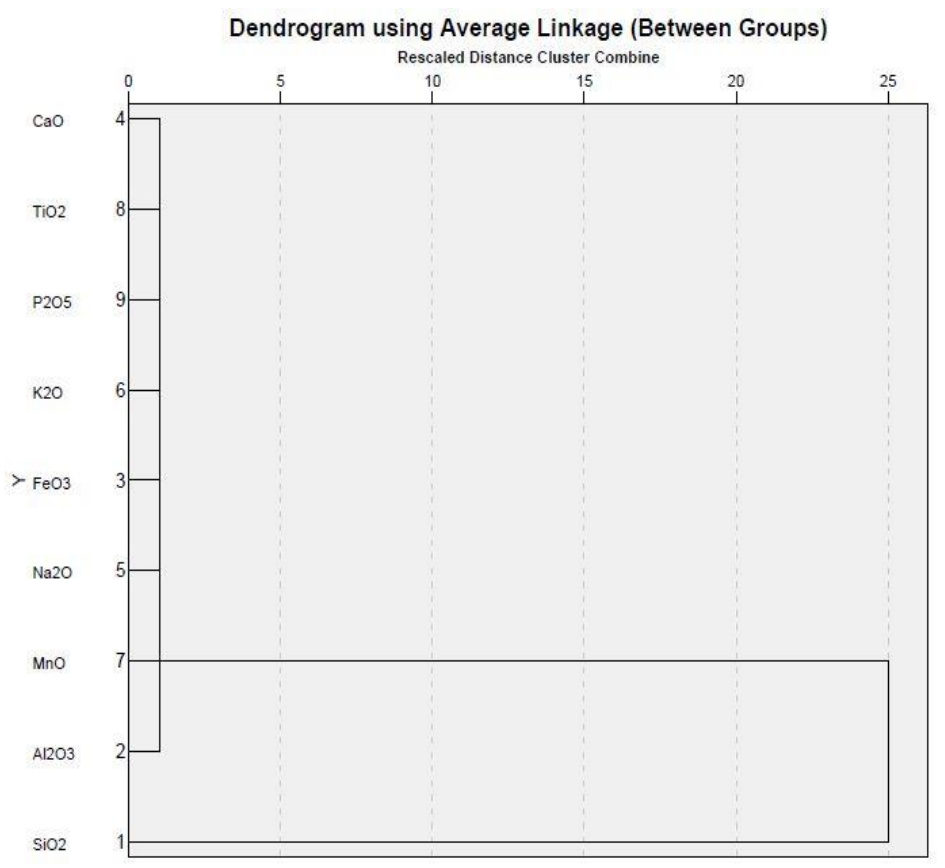

Fig-6: Dendogram image showing clusters of homogenous grouping of Major Oxides in Stream Sediments of Kaiama and environs

The stream sediment data on principal component showed two basic factors with eight stages. The first had ranging from $\mathrm{CaO}-/ \mathrm{TiO}_{2}-\mathrm{P}_{2} \mathrm{O}_{5}-\mathrm{K}_{2} \mathrm{O}-$ $\mathrm{FeO}_{3}-\mathrm{Na}_{2} \mathrm{O}, \mathrm{MnO}, \mathrm{Al}_{2} \mathrm{O}_{3}$ while $\mathrm{MnO}, \mathrm{Al}_{2} \mathrm{O}_{3}$, and $\mathrm{SiO}_{2}$ grouping had the highest loading. This would be regarded as relating to both mineralization and rock weathering of unmineralized rocks that are present in the study area $[23,24]$. 


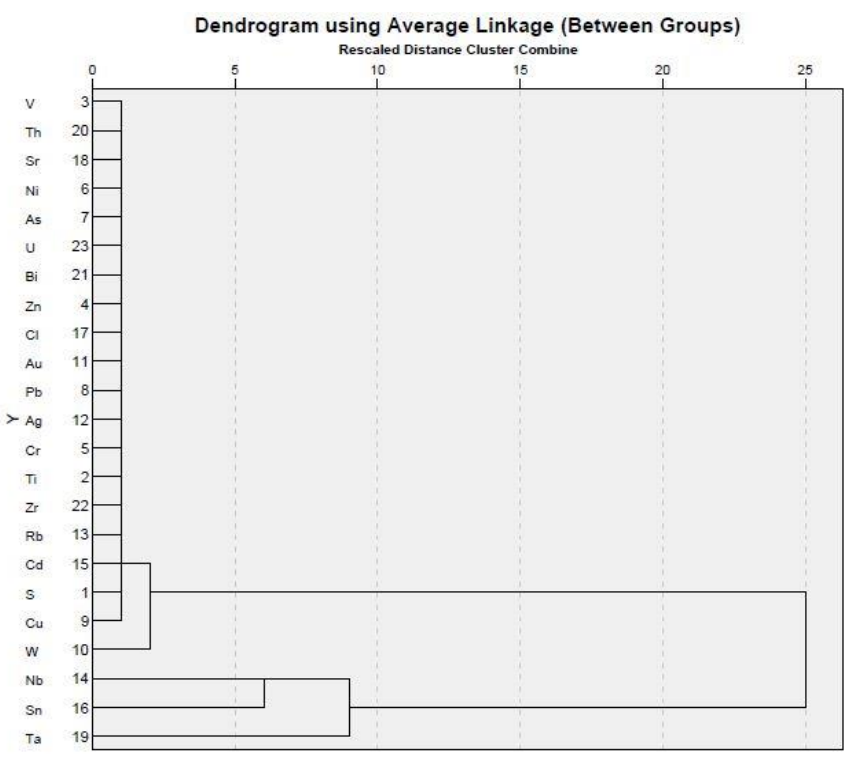

Fig-7: Dendogram image showing clusters of homogenous grouping of Trace elements in quartz/pegmatite veins of Kaiama and environs

In the quartz and pegmatite veins of Kaiama and environs, clustering involved 22 stages which were grouped into five clusters or factors. The first stage is the V, Th, Sr, Ni, As, U, Bi, Zn, Cl and the second cluster $\mathrm{Cd}, \mathrm{S}, \mathrm{Cu}, \mathrm{W}$, the third stage involved $\mathrm{S}, \mathrm{Cu}, \mathrm{W}$ and $\mathrm{Nb}$. The fourth were of $\mathrm{Nb}$ and $\mathrm{S}$, while fifth involved $\mathrm{Nb}, \mathrm{Sn}$ and $\mathrm{Ta}$. The first factor groups relates more to evaporite settings that was rich in Fe - Mn minerals generated from weathering of ferromagnesium rocks with detrital incursion in low oxidizing environment [25-27]. The number of incompatible elements in these factor group is typical of the odd order of geochemical association sedimentary ores [26] while the second, third and fourth factors comprised of $\mathrm{S}, \mathrm{W}, \mathrm{Cd}, \mathrm{Nb}, \mathrm{Sn}, \mathrm{Ta}$ and $\mathrm{Cu}$. These mineralised vein can be said to be made of chalcophile elements because they remain close to the surface and readily combine with sulphur as highly insoluble sulphides

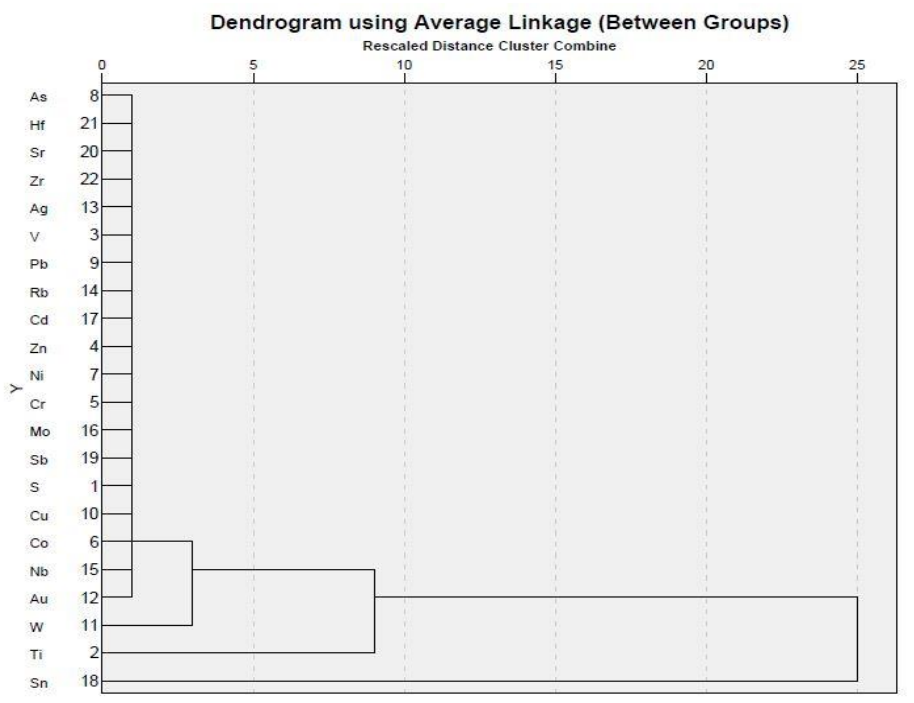

Fig-8: Dendogram image showing clusters of homogenous grouping of Trace elements in Soils of Kaiama and environs

The soil sediment cluster had 21 stages with 4 cluster system. The first consisted of $\mathrm{Hs}, \mathrm{Hf}, \mathrm{Sr}, \mathrm{Zr}, \mathrm{Ag}$, $\mathrm{V}, \mathrm{Pb}, \mathrm{Rb}, \mathrm{Cd}, \mathrm{Zn}, \mathrm{Ni}, \mathrm{Cr}, \mathrm{Mo}, \mathrm{Sb}, \mathrm{S}, \mathrm{Cu}, \mathrm{Co}, \mathrm{Nb}$, and Au. These elements are mostly base metals that are typical of porphyryl copper type system [28]. They also belong to the lithophile elements group of Goldschmidt classification and these elements having significant inter-correlations The second consisted of $\mathrm{Co}, \mathrm{Nb}, \mathrm{Au}$, and $\mathrm{W}$, while the third involved $\mathrm{Nb}, \mathrm{Au}, \mathrm{W}, \mathrm{Ti}$, and the fourth had $\mathrm{Au}, \mathrm{W}, \mathrm{Ti}, \mathrm{Sn}$. Tungsten element groups consisting of $\mathrm{W}, \mathrm{Sn}$, and Ti making up the second cluster system with the highest loading. These are commonly formed in relatively deep environment [29], and belongs to the siderophile element group of Goldsmidt classification. They have high loading density and can be said to be highly translational. 


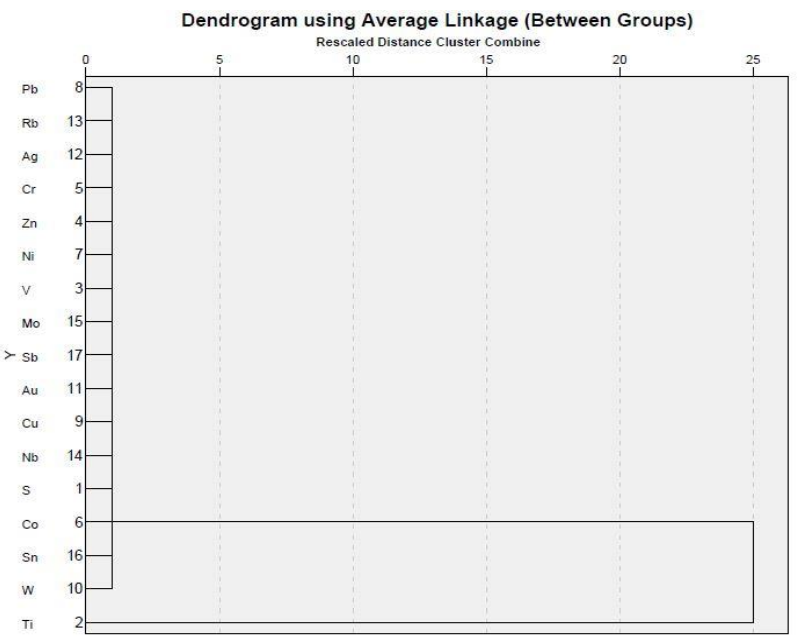

Fig-9: Dendogram image showing clusters of homogenous grouping of trace elements in Stream sediments of Kaiama and environs

These chart majorly divided in to two cluster system comprising of the Base metals from $\mathrm{Pb}-\mathrm{Co}$ interwoven into the tungsten elements group consisting of $\mathrm{W}, \mathrm{Sn}$, and Ti making up the second cluster system with the highest loading. These are commonly formed in relatively deep environment [29], and belongs to the lithophile element group of Goldsmitdt classification.

\section{CONCLUSION}

The trace element geochemical composition shows significant anomalous concentration of $\mathrm{Sn}, \mathrm{W}$, $\mathrm{Ta}, \mathrm{Ti}$, and $\mathrm{Fe}$. $\mathrm{Au}, \mathrm{Cu}$ shows moderate to very high correlation. Areas of significant anomalous concentration were in the 2-4 $\mathrm{km}$ eastern outskirt of Kaiama and as well as 6-10 km south of Kaiama. Sn-W were more to the eastern outskirt while $\mathrm{Sn}$-Ta were more in the southern part of Kaiama.

All other trace elements V, Ni, Sr, Sb, Cd etc. shows low concentration with each other and are not significant in the mineralization of Kaiama. Analytical data for trace elements of Pegmatites from Kaiama were compared with several fields of Ijero-Aramoko, IbadanOshogbo, Oke-Ogun and Kabba-Isanlu. The geochemical data of the trace elements from these fields as acquired by Irvine and Baragat [30], Kuno [31], Okunlola et al., did not conform to the values from Kaiama and environs [32-35].

Further exploration work should be concentrated on soils and stream sediments towards delineating the possible ore deposit in the area of Kaiama and environs.

\section{REFERENCES}

1. Obaje N. G. (2009). Geology and Mineral resources of Nigeria, Springer, Heidelberg, 13-31.

2. Oyinloye, A. O. (2011). Geology and geotectonic setting of the basement complex rocks in South Western Nigeria: implications on provenance and evolution. In Earth and Environmental Sciences. InTech.
3. Kayode, J. S., Nawawi, M. N. M., Abdullah, K. B., \& Khalil, A. E. (2017). Integrating aeromagnetic and Landsat ${ }^{\mathrm{TM}} 8$ data into subsurface structural mapping of Precambrian basement complex. Journal of African Earth Sciences, 125, 202-213.

4. Aliyu, A. S., Musa, Y., Liman, M. S., Abba, H. T., Chaanda, M. S., Ngene, N. C., \& Garba, N. N. (2018). Determination of rare earth elements concentration at different depth profile of Precambrian pegmatites using instrumental neutron activation analysis. Applied Radiation and Isotopes, 131, 36-40.

5. Ogezi, A. E. O. (1977). Geochemistry and Geochronology of Basement Rocks from Northwestern Nigeria. Unpublished Ph.D. Thesis, University of Leeds.

6. Rahaman, M. A. (1988). Recent advances in the study of the basement complex of Nigeria. In: Geological Survey of Nigeria (ed) Precambrian Geology of Nigeria, 11-43.

7. Olade, M. A., \& Elueze, A. A. (1979). Petrochemistry of the Ilesha amphibolite and Precambrian crustal evolution in the Pan-African domain of SW Nigeria. Precambrian Research, 8:303-318.

8. Egbuniwe, I. G. (1982). Geotectonic evolution of the Maru Belt, NW Nigeria. Unpublished Ph.D. Thesis, University of Wales, Aberystwyth.

9. McCurry, P. (1976). The Geology of the Precambrian to Lower Paleozoic rocks of Northern Nigerian, a Review in Kogbe C. A. (Ed.), Lagos, Elizabethan Publishing Co. Geology of Nigeria, 15-39.

10. Rahaman, M. A. (1976). Review of the basement complex of Nigeria in: Kogbe, C. A. (ed) Geology of Nigeria. Elizabethan publishing Lagos, Nigeria, 514.

11. Grant, N. K. (1978). Structural distinction between a metasedimentary cover and an underlying basement in the $600 \mathrm{~m}$.y old Pan-African domain 
of Northwestern Nigeria. Geological Society of America Bulletine, 89: 50-58.

12. Garba, I. (2000). Origin of Pan-African mesothermal gold mineralization of Bin Yauri, Nigeria. Journal of African Earth Sciences, 31(2): 433-449.

13. Danbatta, U. A. (2003). Structural framework underlying the metamorphic evolution of the Kazuare Schist Belt, NW Nigeria. Global Journal of Geological Sciences, 1(1): 82-83.

14. Oluyide, P. O. (1988). Structural Trends in the Nigerian Basement Complex. In: P.O. Oluyide. Precambrian Geology of Nigeria. Geological. Survey of Nigeria, 93-98.

15. Oluyide, P. O., \& Udoh, A. N. (1989). Preliminary comments on the fracture systems of Nigeria. In Proceedings of the National Seminar on earthquakes in Nigeria (Ed.) Ajakaiye D. E, Ojo S. B., \& Daniyan, M. A. 97-109.

16. Ajibade, A. C. (1980). Geotectonic evolution of the Zungeru Region, Nigeria. Unpublished Ph.D. Thesis, University of Wales, Aberystwyth.

17. Danbatta, U. A. (2002). Rb-Sr Isochron Dating of Granitoids from the Kazaure Schist Belts, NW Nigeria. Global journal of Pure and Applied sciences, 8(3): 319-322.

18. Kelly, K. D., \& Hudson, T. (2007). Natural versus anthropogenic dispersion of metals to the environment in the Wulik River area, western Brooks Range, northern Alaska. Geochem: Explor. Environ. Anal, 87-96.

19. Salminen, A., Huuskonen, J., Ojala, J., Kauppinen, A., Kaarniranta, K., \& Suuronen, T. (2008). Activation of innate immunity system during aging: NF-kB signaling is the molecular culprit of inflamm-aging. Ageing research reviews, 7(2), 83105.

20. Allan, A. N., Knut, C., John, Z. P., \& Ahnette, S. (2008). Spatial factor analysis of stream sediments geochemistry data from south Greenland in:Vera Pawlowsky Glahn (ed) proceedings of the third annual conference of the international association for mathematical geology (IAMG 97) www2.1mm.dtu.dk/pubdb/views/edocdownload.php.955-960.

21. Gong, M., Wu, L., Bi, X. Y., Ren, L. M., Wang, L., Ma, Z. D., ... \& Li, Z. G. (2010). Assessing heavy-metal contamination and sources by GISbased approach and multivariate analysis of urban-rural topsoils in Wuhan, central China. Environmental geochemistry and health, 32(1), 59-72.

22. Brian, S. E. (1993). Cluster analysis 3rd Ed. Edward Arnold, London: 169.

23. Bottrill, M. C., Joseph, L. N., Carwardine, J., Bode, M., Cook, C., Game, E. T., ... \& Pressey, R.
L. (2008). Is conservation triage just smart decision making?. Trends in ecology \& evolution, 23(12), 649-654.

24. Bamigboye, O. S., \& Adekeye, J. I. D. (2011). Stream sediment survey of Eruku and its environs, Central Nigeria: implication for exploration. International Journal of Research and Reviews in Applied Sciences, 7(2), 160-171.

25. McQueen, K. G. (2004). Ore deposit types and their expressions. Regolith expression of Australian ore systems, edition: 1, 1-14

26. Krauskopf K. B., \& Bird, D. K. (1985). Introduction to Geochemistry. McGraw-Hill Educaton (ISE Edition).

27. Levinson, L. M. (1981). Mossbauer spectroscopy for pyrite analysis in coal. In: Chemistry and Physics of Coal Utilization - 1990, AIP Conference Proceedings No. 70, B.R. Cooper and L. Petrakis, eds. American Institute of Physics, New York, 209-234.

28. Seedorf, E., Dilles, J. H, Profett, J. M., \& Barton, M. (2005): Porphyry deposits: Characteristics and Origin of Hypogene features. In: Economic Geology $100^{\text {th }}$ Anniversary. 251-298.

29. Einaudi, M. T., Meinart, L. D., \& Newberry, R. J. (1981). Skarn Deposits: Economic Geology, 75th Anniv. 317-391.

30. Irvine, T. N., \& Baragar, W. R. A. (1971). A guide to chemical classification of common Volcanic rocks. Journal of earth sciences, 8(5):523-548.

31. Kuno, H. (1968). Differentiation of Basalt Magmas. In: Basalts, Ed. Hess, H. H., \& Poldervaart, A. Interscience Publisher, 2, 623-686

32. Okunlola, O. A. (2001). Geological and compositional investigation of Precambrian marble bodies and associated rocks in the Burum and Jakura areas, Nigeria. Unpublished Ph. D. thesis.

33. Okunola, O. A., \& Ocan, O. O. (2002). The expected environmental impact and mitigation studies of organized mining of rare metal (Ta-Sn$\mathrm{Nb})$ pegmatites around Keffi area. Northcentral Nigeria. Journal Environmental Extension.., 3, 6468.

34. Okunlola, O. A. (2005). Metallogeny of TantalumNiobium Mineralization of Precambrian Pegmatites of Nigeria. Mineral Wealth, 137, 3850.

35. Okunlola, A. O., \& Jimba, S. (2006). Compositional Trends in Relation to TantalumNiobium Mineralization in Precambrian pegmatites of Aramoko-Ara-Ijero Area, Southwestern Nigeria. Journal of Mining and Geology, 42: 113-126. 